\title{
Parametric spherical deconvolution: Inferring anatomical connectivity using diffusion MR imaging
}

\author{
Enrico Kaden, ${ }^{*}$ Thomas R. Knösche, and Alfred Anwander \\ Max Planck Institute for Human Cognitive and Brain Sciences, Stephanstr. 1a, 04103 Leipzig, Germany
}

Received 21 September 2006; revised 19 April 2007; accepted 7 May 2007

Available online 18 May 2007

\begin{abstract}
The human brain forms a complex neural network with a connectional architecture that is still far from being known in full detail, even at the macroscopic level. The advent of diffusion MR imaging has enabled the exploration of the structural properties of white matter in vivo. In this article we propose a new forward model that maps the microscopic geometry of nervous tissue onto the water diffusion process and further onto the measured MR signals. Our spherical deconvolution approach completely parameterizes the fiber orientation density by a finite mixture of Bingham distributions. In addition, we define the term anatomical connectivity, taking the underlying image modality into account. This neurophysiological metric may represent the proportion of the nerve fibers originating in the source area which intersect a given target region. The specified inverse problem is solved by Bayesian statistics. Posterior probability maps denote the probability that the connectivity value exceeds a chosen threshold, conditional upon the noisy observations. These maps allow us to draw inferences about the structural organization of the cerebral cortex. Moreover, we will demonstrate the proposed approach with diffusion-weighted data sets featuring high angular resolution.

(c) 2007 Elsevier Inc. All rights reserved.
\end{abstract}

Keywords: Parametric spherical deconvolution; Finite mixture of Bingham distributions; Anatomical connectivity; Bayesian statistics; Diffusion MR imaging

\section{Introduction}

It is widely accepted that specialization is a fundamental property of brain organization, involving the segregation and integration of neural populations (Zeki and Shipp, 1988). These discrete cortical areas are connected by long-range nerve fibers which shorten the path of neural information processing, enabling the rapid interaction of distributed brain modules. The low processing depth necessitates highly parallel computing facilities made up of a large number of connections that are neither wellordered nor completely random (Sporns et al., 2000), but are

\footnotetext{
* Corresponding author.

E-mail address: kaden@cbs.mpg.de (E. Kaden).

Available online on ScienceDirect (www.sciencedirect.com).
}

grouped into fiber bundles. However, detailed knowledge about the connectional architecture of white matter is still rather limited even at the macroscopic level, not least due to the anatomical variability between subjects. With the advent of diffusion magnetic resonance (MR) imaging, it has become possible to study the complex system of long-range nerve fibers in a non-invasive way (Le Bihan, 2003). This unique method measures the Brownian motion of water molecules in different directions in the underlying material. The microscopic tissue structures hinder the diffusing molecules, which thereby exhibit anisotropic diffusion patterns when the observation period is chosen to be sufficiently long. Although the nerve fibers cannot be observed directly, the directional dependence of water diffusion seems to be primarily caused by the coherent orientation of the fibers within a microscopic environment, providing a basis for the reconstruction of fiber pathways.

To obtain information on the course of fiber tracts and the degree of the connectedness of distant brain areas, we need to construct a forward model that maps the microgeometry of nervous tissue onto the diffusion process and further onto the measured MR signals. Since the architecture of neural cells is highly complex, there is no doubt about the necessity to make assumptions and simplifications. Also, the number of diffusion encoding gradients is limited and we typically measure no more than one $b$-value in addition to $b=0$, which is required by the normalization of the $T_{2}$ relaxation. Usually, fiber bundles are mapped onto diffusionweighted MR signals by the diffusion tensor model proposed by Basser et al. (1994). This approach proved inadequate for describing crossings and branchings of nerve fiber tracts, but it is fairly straightforward to represent several fiber bundles by a sum of multiple diffusion tensors weighted by their respective volume fractions (Tuch et al., 2002; Parker and Alexander, 2003; Hosey et al., 2005). However, a Taylor expansion at $b=0$ suggests that the observed data will not provide enough information to resolve the water diffusivity and the volume fraction for small $b$-values independently (Basser and Jones, 2002). For that reason, the multiple tensor model is frequently constrained, using conditions such as rotationally symmetric tensors, constant diffusivities or fixed volume fractions. These constraints do not form any natural invariant. For instance, the diffusion properties of a fiber bundle also encode the spreading of the fibers within that bundle. This 
spreading is far from being constant throughout the white matter. The uncertainty about the orientation of the fiber bundles in those (multiple) tensor models might be described by Watson or Bingham distributions (Basser and Pajevic, 2000; Cook et al., 2004, 2005). There have been a great many alternative suggestions dealing with complex fiber structures. As the focus of this article is on explicit forward models, we ignore all methods which take only the diffusion propagator or other derived functions (Tuch, 2004; Wedeen et al., 2005; Özarslan et al., 2006) into account. For those models and other approaches, see the recent review by Le Bihan (2003) and the references therein.

In this work we use the general idea of spherical deconvolution (Behrens et al., 2003; Tournier et al., 2004; Anderson, 2005) to model the primary structural properties of nervous tissue. Consider a population of nerve fibers within a microscopic environment and assume that the diffusion characteristics of a plain fiber as the elementary component are invariant throughout the brain. The spherical convolution of the fiber orientation density with the specified diffusion response function of a fiber segment yields the diffusion propagator and then the measured MR signals. Tournier et al. (2004) and Anderson (2005) proposed the use of Fourier analysis on the sphere. Representation by spherical harmonics, which form a complete orthonormal basis over the squareintegrable functions on the sphere, reduces the deconvolution of the fiber orientation density to multiplications (Healy and Kim, 1996). Since in general spherical harmonics do not ensure that the density function is non-negative and normalized, Jansons and Alexander (2003) and more recently Alexander (2005a) determined the fiber orientation density by a maximum entropy approach. In contrast to the above authors, we propose an explanatory parameterization of the fiber orientation density, which allows us to determine the number of fiber bundles and their orientation and volume fraction.

To describe the complex connectivity patterns in the human brain, we need a measure quantifying the connectedness of two cortical areas by means of nerve fibers that, on the one hand, limits itself to the type of information that is provided by diffusionweighted MR data sets (e. g., it does not make any sense to distinguish afferent from efferent fiber pathways) and, on the other hand, has a clear neurophysiological meaning. Obviously, different experimental modalities might lead to different definitions of the term anatomical connectivity. Preliminary work includes the connectivity measure proposed by Koch et al. (2002), who determined the transition probability from a voxel to its adjacent voxels. These authors then estimated the structural connectivity by calculating the proportion of the random walks originating in the source voxel that intersect a given target region while moving throughout the voxel lattice. To avoid the errors due to the discretization of the transition probability, Parker et al. (2003) and, in the case of multiple fiber bundles, Parker and Alexander (2003) continuously solved the curve integral of a fiber streamline, whose starting point is usually the center of a voxel, and obtained the Probabilistic Index of Connectivity (PICo), which is closely related to the aforementioned metric by Koch et al. (2002). Behrens et al. (2003) defined the anatomical connectivity as the probability that there exists a fiber pathway which links a specified point with the target region. This raises the question of whether it is reasonable to ask for the existence of such a particular fiber when normally considering voxels, i.e. start regions that have a volume greater than zero. Since the above approaches do not allow the choice of initial regions different from isolated points, we propose to generalize the definition of the term anatomical connectivity, thereby dissociating between the neurobiological quantity and the probability thereof.

The specified inverse problem is, decomposed into simpler subproblems, tackled using Bayesian statistics (Behrens et al., 2003), which provides a parametric inferential framework for statistical modeling and analysis. In the following, the Theory and methods section introduces the novel forward model that exposes the nerve fiber population voxel by voxel. With the addition of the prior distribution of the model parameters, we obtain a complete Bayesian problem for the estimation of the fiber orientation density conditional upon the noisy MR measurements. The global model component then reconstructs the nerve fiber pathways and estimates the posterior distribution of the anatomical connectivity between arbitrarily chosen cortical regions. In Appendix A we deal with the computational issues of Bayesian inference. The Results section includes simulations of the parametric spherical deconvolution and exemplifies a connectivity analysis concerning the crossing of the callosal fibers and the corona radiata using diffusion-weighted data sets featuring high angular resolution. We conclude with a discussion of the proposed models.

\section{Theory and methods}

\section{Parametric spherical deconvolution}

Brownian motion is the random, thermally driven self-movement of molecules (Einstein, 1905). Consider a population of water molecules providing a spin ensemble within a microscopic environment of nervous tissue. The diffusion propagator $\pi(\boldsymbol{R} \mid \Delta)$ states the ensemble-averaged probability that a spin moves through displacement $\boldsymbol{R}$ during the time interval $\Delta$. The pulsed gradient spin echo experiment (Stejskal and Tanner, 1965) enables the measurement of this diffusion process. Let $b=\Delta|\boldsymbol{q}|^{2}$ be the diffusion weighting factor and $\boldsymbol{g}=\boldsymbol{q} /|\boldsymbol{q}|$ the normalized diffusion encoding gradient direction (with the scattering wave vector $\boldsymbol{q}$ ). Under the narrow-pulse approximation we obtain the Fourier transform (Callaghan, 1993)

$\frac{E_{\Delta}(\boldsymbol{q})}{E_{\Delta}(\mathbf{0})}=\int \underset{\mathbb{R}^{3}}{\pi}(\boldsymbol{R} \mid \Delta) \exp (i \boldsymbol{q} \cdot \boldsymbol{R}) d \boldsymbol{R}$

where $E_{\Delta}(\boldsymbol{q})$ is the spin echo signal in the presence of the diffusion weighting $\boldsymbol{q}$ and $E_{\Delta}(\mathbf{0})$ denotes the MR signal in the absence of any diffusion weightings.

\section{Forward model}

The above diffusion propagator $\pi(\boldsymbol{R} \mid \Delta)$ does not only depend on the diffusion time $\Delta$, but also on the microgeometrical properties of the tissue under consideration. The axonal membranes of the nerve fibers, which are densely packed in the white matter of the brain and spinal cord, seem to form the structural component that mainly determines the anisotropic water diffusion (Beaulieu, 2002). Consider a population of nerve fibers within a voxel. We distinguish three levels of description: a single fiber, a fiber subpopulation (also called a fiber bundle), and the entire fiber population. The main idea is to discretize the fiber population into a finite number of subpopulations, which is a priori unknown, using the criterion that the fibers within a subpopulation should be coherently oriented. The associated missing data problem dealing 
with the assignment of a particular fiber to a fiber bundle (Marin et al., 2005) will not be elaborated any further here.

The diffusion process in a single fiber segment (which should also include its typical immediate surroundings outside the axonal membranes) is assumed to be governed by a trivariate Gaussian distribution $\mathcal{N}(\mathbf{0}, 2 \Delta \boldsymbol{D})$ with a mean of $\mathbf{0}$ and the 3-by-3 symmetric positive-definite covariance matrix $2 \Delta \boldsymbol{D}$. The geometric tissue properties determine the eigenstructure of the apparent diffusion tensor $\boldsymbol{D}$. The eigenvector $\omega \in S^{2}$ corresponding to the largest eigenvalue $\lambda_{1}$ denotes the fiber orientation where $S^{2}=$ $\left\{x \in R^{3}:\|x\|=1\right\}$ is the two-dimensional unit sphere. The underlying assumption is that the water diffusivity is highest parallel to the nerve fibers. The (myelinated) cell membranes perpendicular to the fiber direction provide the greatest barrier to diffusion. Since small fiber sections have an approximately cylindrical geometry, it is reasonable to assume that the transverse water diffusion is rotationally symmetric and thus the two smaller eigenvalues $\lambda_{2}=\lambda_{3}$ are equal. The inversion of the eigen-decomposition yields

$\boldsymbol{D}(\omega)=\left(\lambda_{1}-\lambda_{2}\right) \omega \omega^{t}+\lambda_{2} \boldsymbol{I}_{3}$

with a fiber orientation $\omega$ and apparent diffusion coefficients $\lambda_{1} \geq \lambda_{2} \geq 0$ where $\boldsymbol{I}_{3}$ is the three-dimensional unit matrix.

As the nerve fibers, which form the elementary components of a fiber population, have similar dimensions, we suppose that their diffusion characteristics $\left\{\lambda_{1}, \lambda_{2}\right\}$ are constant throughout the white matter of the brain. Therefore, the diffusion propagator $f_{\mathcal{N}}(\boldsymbol{R} ; \mathbf{0}, 2 \Delta \boldsymbol{D}(\omega))$ for a fiber segment only depends on its orientation $\omega \in S^{2}$. If the fiber direction is described by a density function $p(\omega)$, the spherical continuous mixture model

$\pi(\boldsymbol{R} \mid \Delta)=\int_{S^{2}} p(\omega) f_{\mathcal{N}}(\boldsymbol{R} ; \mathbf{0}, 2 \Delta \boldsymbol{D}(\omega)) d \omega$

with the constraints

$0 \leq p(\omega)$ and $\int_{S^{2}} p(\omega) d \omega=1$

yields the propagator for the entire fiber population. Since we focus on the structural properties of nervous tissue, the objective is to estimate the function $p$. There are two different approaches by which to proceed. The non-parametric strategy attempts to find $p$ in a specified function space (Tournier et al., 2004; Anderson, 2005; Alexander, 2005a). The other approach, which we adopt here, parameterizes the fiber orientation density.

First, we assume that the fiber population consists of one fiber bundle. Since the diffusion propagator of a fiber segment is antipodally symmetric, the fiber orientation density $p$ is set to be antipodally symmetric, i. e. $p(\omega)=p(-\omega)$. We propose to model $p(\omega)$ through a Bingham distribution (Bingham, 1974), which is defined as

$f_{\mathcal{B}}(\omega ; \boldsymbol{B})=\frac{1}{c_{\mathcal{B}}(\boldsymbol{B})} \exp \left(\omega^{t} \boldsymbol{B} \omega\right)$

where $\boldsymbol{B}$ is a 3 -by-3 symmetric matrix and $c_{\mathcal{B}}(\boldsymbol{B})$ denotes the normalizing constant (see Appendix A.1). The Bingham density $f_{\mathcal{B}}(\omega ; \boldsymbol{B})$ can be regarded as a trivariate Gaussian distribution conditional upon the two-dimensional unit sphere, which is invariant with respect to the addition of an arbitrary constant to the eigenvalues of $\boldsymbol{B}$. The eigenstructure of $\boldsymbol{B}$ describes the arrangement of the fibers. The eigenvector $\mu$ corresponding to the largest eigenvalue $\kappa_{1}$ represents the fiber bundle orientation. Its sign is ambiguous. Without a loss of generality, let be $\kappa_{1} \geq \kappa_{2} \geq \kappa_{3}$ where $\kappa_{2}$ and $\kappa_{3}$ are the other two eigenvalues. The differences $\kappa_{1}$ $-\kappa_{2}$ and $\kappa_{2}-\kappa_{3}$ quantify the (asymmetric) spreading of the nerve fibers around the axis $\mu$ (Fig. 1). In particular, the proposed parameterization of $p$ allows for the modeling of fiber bundles of sheetlike appearance. However, a voxel does not necessarily contain only one fiber bundle. There are voxels that are composed of $N$ approximately homogeneous fiber subpopulations which may have different orientations. We therefore extend the above parameterization of the fiber orientation density by considering the finite mixture of Bingham distributions

$p(\omega)=\sum_{i=1}^{N} P_{i}^{\prime} f_{\mathcal{B}}\left(\omega ; \boldsymbol{B}_{i}\right)$

with the volume fractions $0 \leq P_{i}{ }^{\prime}$ and $\sum_{i=1}^{N} P_{i}{ }^{\prime}=1$ for the fiber bundles $i=1, \ldots, N$. The fiber population is thus discretized into $N$ subpopulations where the eigenvector corresponding to the largest eigenvalue of $\boldsymbol{B}_{i}$ denotes the mean orientation of the nerve fibers
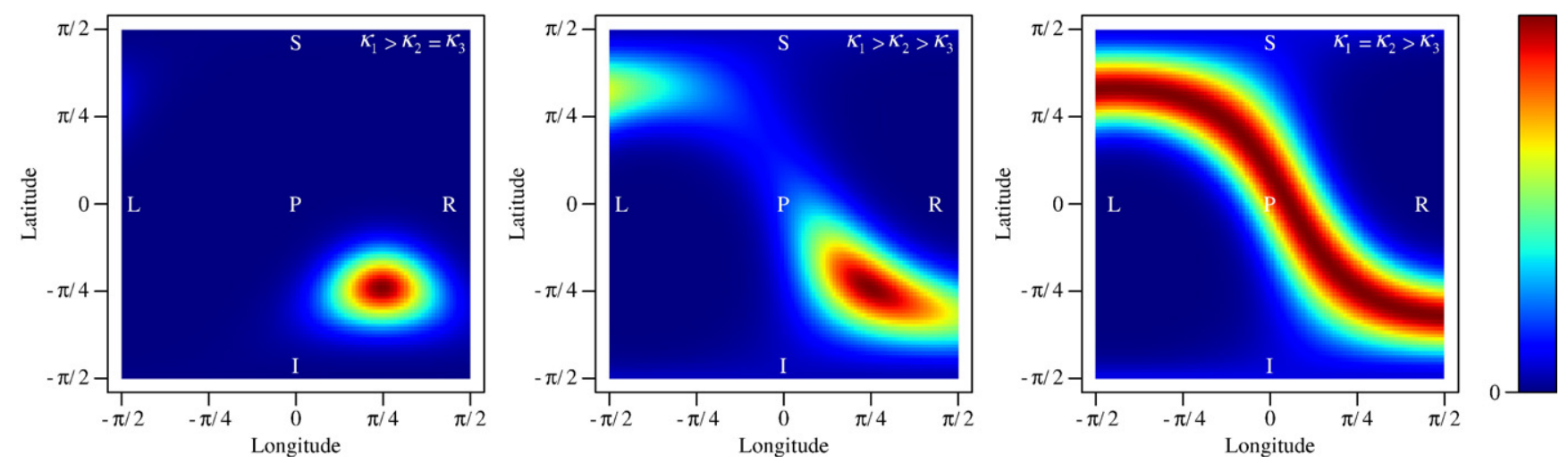

Fig. 1. The Bingham distribution models the orientation density function of the nerve fibers within a bundle. We can differentiate four cases as follows (from left to right). If the eigenvalues $\kappa_{1}=\kappa_{2}=\kappa_{3}$ are equal, the direction of the fibers is uniformly distributed on the sphere, which is not shown. In case of $\kappa_{1}>\kappa_{2}=\kappa_{3}$, the arrangement of the nerve fibers is rotationally symmetric and bipolar. If $\kappa_{1}>\kappa_{2}>\kappa_{3}$, we have a Bingham distribution which is not a Watson density, since the fibers are not rotationally symmetrically oriented. Finally, in case of $\kappa_{1}=\kappa_{2}>\kappa_{3}$, the distribution of the fibers is rotationally symmetric and equatorial around the eigenvector associated with $\kappa_{3}$. Abbreviations: inferior (I), superior (S), left (L), right (R), posterior (P). See text for further details. 
within the $i$ th subpopulation. Note that the constraints formulated in Eq. (4) are fulfilled.

Human brain white matter is composed of (myelinated) axons and glial cells. Therefore, an additional variable is considered that describes the volume fraction of the space between the nerve fibers and thus their packing density. We assume that the glial cells and the extra-cellular space only contribute to isotropic water diffusion, which is governed by a trivariate Gaussian density $\mathcal{N}\left(\mathbf{0}, 2 \Delta \lambda_{0} \boldsymbol{I}_{3}\right)$ with a mean of $\mathbf{0}$ and an apparent diffusion coefficient of $\lambda_{0}$. This compartment is additively incorporated into the diffusion propagator $\pi(\boldsymbol{R} \mid \Delta)$ weighted by its volume fraction $0 \leq P_{0} \leq 1$. Now we complete the forward model. The diffusion propagator

$$
\begin{aligned}
\pi(\boldsymbol{R} \mid \Delta)= & P_{0} f_{\mathcal{N}}\left(\boldsymbol{R} ; \mathbf{0}, 2 \Delta \lambda_{0} \boldsymbol{I}_{3}\right) \\
& +\int_{S^{2}}\left(\sum_{i=1}^{N} P_{i} f_{\mathcal{B}}\left(\omega ; \boldsymbol{B}_{i}\right)\right) f_{\mathcal{N}}(\boldsymbol{R} ; \mathbf{0}, 2 \Delta \boldsymbol{D}(\omega)) d \omega
\end{aligned}
$$

with $P_{i}=\left(1-P_{0}\right) P_{i}^{\prime}$ for $i=1, \ldots, N$ is defined in dependence of the underlying tissue architecture $\left\{N, P_{0},\left(P_{i}, \boldsymbol{B}_{i}\right)_{i=1, \ldots, N}\right\}$. Inserting this diffusion propagator into the Fourier transform (1) and substituting $\boldsymbol{D}(\omega)$ according to Eq. (2), we obtain the parametric spherical convolution model (for $N>0$ )

$$
\begin{aligned}
\frac{E_{\Delta}(\boldsymbol{q})}{E_{\Delta}(\mathbf{0})}= & P_{0} \exp \left(-\Delta|q|^{2} \lambda_{0}\right)+\exp \left(-\Delta|q|^{2} \lambda_{2}\right) \\
& \times \sum_{i=1}^{N} P_{i} \frac{{ }_{1} \mathrm{~F}_{1}\left(1 / 2 ; 3 / 2 ; \boldsymbol{B}_{i}-\Delta\left(\lambda_{1}-\lambda_{2}\right) \boldsymbol{q} \boldsymbol{q}^{t}\right)}{{ }_{1} \mathrm{~F}_{1}\left(1 / 2 ; 3 / 2 ; \boldsymbol{B}_{i}\right)}
\end{aligned}
$$

which generates the observable MR signals. For the derivation of this spherical convolution approach, see Appendix A.2, where further special cases are considered. If the fiber population cannot be discretized into distinct fiber bundles $(N=0)$, the model becomes

$$
\begin{aligned}
\frac{E_{\Delta}(\boldsymbol{q})}{E_{\Delta}(\mathbf{0})}= & P_{0} \exp \left(-\Delta|\boldsymbol{q}|^{2} \lambda_{0}\right)+\left(1-P_{0}\right) \exp \left(-\Delta|\boldsymbol{q}|^{2} \lambda_{2}\right) \\
& \times{ }_{1} \mathrm{~F}_{1}\left(1 / 2 ; 3 / 2 ;-\Delta|\boldsymbol{q}|^{2}\left(\lambda_{1}-\lambda_{2}\right)\right)
\end{aligned}
$$

where the fiber direction is uniformly distributed on the sphere. The macroscopic diffusion process of the whole fiber population is isotropic, though the water diffusion in the nerve fibers is strictly anisotropic. The crucial point to note is that the parameterization (5) of the fiber orientation density yields a closed form. The hypergeometric function ${ }_{1} \mathrm{~F}_{1}(1 / 2 ; 3 / 2 ;$.) of a matrix argument (Herz, 1955 ) may be computed by a saddlepoint approximation (Kume and Wood, 2005).

\section{Bayesian inference}

The inverse problem of estimating the structural tissue properties from the water diffusion process is solved by Bayesian statistics, in a similar way to Behrens et al. (2003). This approach enables us to analyze both the model parameters and the adequacy of the models proposed to explain an observed phenomenon. The prior knowledge about the parameters $\theta$, which include the MR signal $E_{\Delta}(\mathbf{0})$ without any diffusion weightings and the estimation error $\sigma^{2}$, is encoded in the probability distribution $\pi\left(\theta \mid m_{N}\right)$. The likelihood function $f\left(z ; \theta, m_{N}\right)$ that updates $\pi\left(\theta \mid m_{N}\right)$ by extracting the information on the hidden parameters $\theta$ from the observations $z$ is formed by the forward model $m_{N}$ with $N$ fiber bundles, which is formulated in Eqs. (6) and (7), under the assumption that the measured magnitude of the complex spin echo signal follows a Rician distribution (Sijbers and den Dekker, 2004). The fiber bundles in neighboring voxels are implicitly supposed to be independently oriented. According to Bayes' theorem, we obtain the posterior distribution $\pi\left(\theta \mid z, m_{N}\right) \propto f\left(z ; \theta, m_{N}\right) \pi\left(\theta \mid m_{N}\right)$ which reflects the uncertainty about $\theta$ after we have seen the measured data $z$. The posterior distribution is only known up to a normalizing constant, which does not exist in analytic form. Therefore, we simulate $\pi\left(\theta \mid z, m_{N}\right)$ using Markov chain Monte Carlo methods (see Appendix A.3).

We choose weakly-informative prior distributions that make as few assumptions about the hidden parameters $\theta$ as possible. The eigen-decomposition of the symmetric matrix $\boldsymbol{B}_{i}$ representing the fiber directions within the $i$ th bundle yields $\boldsymbol{B}_{i}=\boldsymbol{R}_{i} \operatorname{diag}\left(\kappa_{1, i}, \kappa_{2, i}\right.$, $\left.\kappa_{3, i}\right) \boldsymbol{R}_{i}^{-1}$. The rotation matrix $\boldsymbol{R}_{i}$ may be reformulated by the Euler angles $\left(\phi_{i}, \theta_{i}, \psi_{i}\right)$. Since the orientation of the $i$ th fiber subpopulation is supposed to be uniformly distributed on the twodimensional unit sphere, we set

$\left(\phi_{i}, \theta_{i}, \psi_{i}\right) \sim \frac{1}{2} \sin \left(\theta_{i}\right) \mathbb{I}_{[0, \pi)}\left(\theta_{i}\right)$.

$\mathbb{I}_{[0, \pi)}\left(\theta_{i}\right)$ is the indicator function that is 1 if $\theta_{i} \in[0, \pi)$ and otherwise 0 . As the addition of an arbitrary constant to the eigenvalues of $\boldsymbol{B}_{i}$ does not change the Bingham distribution, we assume that

$\kappa_{1, i}, \kappa_{2, i}, \kappa_{3, i} \sim \mathcal{N}\left(0, \sigma_{\kappa}\right)$

with the constraint $\kappa_{1, i} \geq \kappa_{2, i} \geq \kappa_{3, i}$, where $\sigma_{\kappa}$ is an appropriately chosen hyperparameter. The volume fractions should follow the Dirichlet distribution

$\left(P_{0}, P_{1}, \ldots, P_{N}\right) \sim \mathcal{D}(1,1, \ldots, 1)$.

For the true magnitude of the signal in the absence of any diffusion weightings with the observation period $\Delta$, we set

$\left.E_{\Delta}(\mathbf{0}) \sim \mathcal{U}_{[0,} E_{\max }\right]$

where $E_{\max }$ is a sufficiently high value covering the whole finite range of $T_{2}$-weighted MR signals.

Next, we address the issue of establishing the prior distribution of the estimation error $\sigma^{2}$. The MR measurements are corrupted by thermal noise caused by random fluctuations in the receiving coil electronics and the sample material under examination (Haacke et al., 1999). The parameter $\tau^{2}$ characterizing the noise variance of the observed magnitude signal, which should follow a Rician distribution as pointed out above, is supposed to be stationary throughout the MR volume. Masking a background region $\Omega$ with an unknown but constant signal, the posterior distribution $\pi\left(\tau^{2} \mid \Omega\right)$ is estimated from $\Omega$ in the raw data set via Bayesian inference. The estimation error $\sigma^{2}$ is composed of both the thermal noise inherent in MR measurements and the inadequacies of the specified model. However, the observed estimation error can be smaller than the error one would expect considering the thermal noise $\tau^{2}$. This is due to an overfitting of the model. Therefore, we set the prior distribution of the estimation error to

$\left.\sigma^{2} \sim \mathcal{I} \mathcal{G}(\alpha, \beta)\right|_{[Z, \infty)}, \quad Z \sim \pi\left(\tau^{2} \mid \Omega\right)$ 
where $\left.\mathcal{I} \mathcal{G}(\alpha, \beta)\right|_{[\gamma, \infty)}$ is an inverse Gamma density with appropriately chosen hyperparameters $\alpha$ and $\beta$ that is restricted to the interval $[\gamma, \infty)$. It is worth mentioning that we use only proper prior distributions.

The number of fiber bundles $N$ in a given voxel is a priori unknown. The Bayesian paradigm enables the selection of the model $m_{N}$ that best explains the observed phenomenon without adding unnecessary complexity, thereby differentiating between the fiber subpopulations and the noise present in MR measurements. In the previous subsection we have specified a collection of alternative forward models $m_{i}$ consisting of $i=0,1, \ldots, N_{\max }$ fiber bundles where $N_{\max }$ denotes the maximum number we want to consider. Without a loss of generality, let $m_{i}$ and $m_{j}$ be two models with $i>j$. The Bayes factor (e. g., Hosey et al., 2005) is defined by $\xi_{i j}=\pi\left(z \mid m_{i}\right) / \pi\left(z \mid m_{j}\right)$ where $\pi\left(z \mid m_{k}\right)=\int \pi\left(z \mid \theta_{k}, m_{k}\right) \pi\left(\theta_{k} \mid m_{k}\right) \mathrm{d} \theta_{k}$ denotes the predictive probability that the model $m_{k}$ for $k=i, j$ generates the measured data $z$ (Kass and Raftery, 1995). Newton and Raftery (1994) proposed the harmonic mean estimator to approximate $\pi\left(z \mid m_{k}\right)$. Then $\xi_{i j}$ states the evidence that the model $m_{i}$ is preferred to the model $m_{j}$. According to Occam's Razor, we choose the simpler model $m_{j}$, i. e. the model with the smaller number of fiber bundles, except if there is strong evidence in favor of the model $m_{i}$. Jeffreys (1961) assumed decisive evidence if the Bayes factor $\xi_{i j}$ exceeds 100 .

\section{Anatomical connectivity}

In order to study the issue of connectional neuroanatomy in a rigorous manner, we need a measure that quantifies the connectivity between two given cortical areas. After the nerve fiber populations have been estimated voxel by voxel, the global model component reconstructs the fiber pathways and addresses the definition of the term anatomical connectivity.

\section{Fiber tractography}

To begin with, we assume that the number of fiber bundles in every voxel is $N=1$. Fiber tractography is performed on a sample drawn from the posterior distribution of this simplified fiber orientation field, which is inferred from the noisy data via Bayesian statistics. Note that diffusion MR imaging allows us to observe the orientation of fiber segments, but not their location within a voxel. The only spatial encoding comes from the position and the size of a voxel, which has a volume greater than zero. Due to this lack of spatial information, the fiber pathways cannot be immediately reconstructed, since the precise tangent vector $\omega(\boldsymbol{x})$ of a nerve fiber at the specified point $\boldsymbol{x} \in \mathbb{R}^{3}$ is not known, as exemplified in Fig. 2. We only know the relative frequency of an orientation in the voxel containing $\boldsymbol{x}$, which is implemented by the fiber orientation density. In this work we adopt the simple approach that the orientation at the center of a voxel is assumed to correspond to the fiber bundle orientation, which is by definition the mean orientation of the fiber sections within a bundle. Then $\omega(\boldsymbol{x})$ at an arbitrarily chosen $\boldsymbol{x}$ is estimated by trilinear interpolation, thereby considering the fiber population in the neighboring voxels. Let $x_{0}$ be the starting point of a nerve fiber. The fiber streamline $c\left(x_{0}\right)$ is determined by solving a curve integral on the resulting vector field (Conturo et al., 1999; Mori et al., 1999). This initial value problem is numerically computed by the fourth-order Runge-Kutta algorithm. The sign of the fiber direction is chosen such that the local curvature of the fiber tract is not greater than $90^{\circ}$. The linear interpolation could be replaced by a weighted interpolation scheme that makes use of the
Spatial and orientation encoding

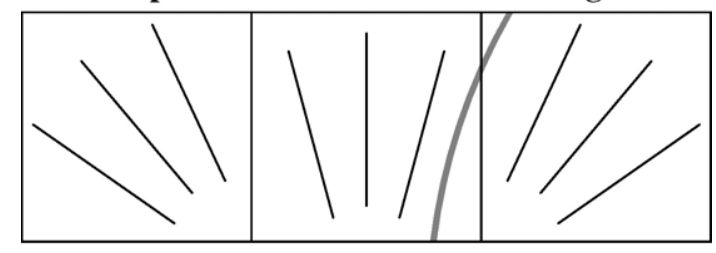

Only orientation encoding

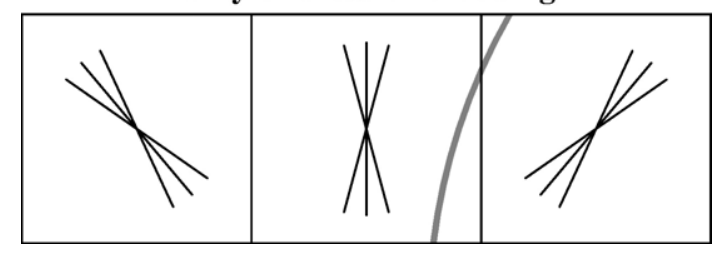

Fig. 2. The diffusion-weighted MR signals and consequently the fiber orientation density do not include any information about the location of the nerve fibers within a voxel (bottom). The spatial relationship of the fiber segments is established by the tractography algorithm that considers the fiber populations in the neighboring voxels (top). In particular, the topological relationship of the nerve fibers within a bundle depending on the direction in which the fiber bundle fans is preserved. The reconstruction of the fiber pathway shown in gray color is based on the assumption that the nerve fiber system meets certain continuity conditions.

fiber spreading, which is modeled by Bingham densities. Alternatively, a probabilistic tractography algorithm (e. g., Friman et al., 2006) might be used that samples the Bingham distributions to obtain the tangential direction of the nerve fibers.

Next, to deal with crossing nerve fibers, we generalize the aforementioned vector field to the fiber orientation field which assigns the number of fiber bundles $N$ as well as the volume fraction $P_{i}$ and orientation $\left(\theta_{i}, \phi_{i}\right)$ of the $i$ th fiber subpopulation (for $i=1, \ldots, N$ ) to every voxel within the white matter. Since the fiber population is represented by a finite mixture of Bingham densities and the components of this mixture are invariant with respect to the permutation of indices, an identifiability problem, which selects the corresponding fiber bundle at each voxel, has to be overcome. A straightforward approach is to choose that fiber subpopulation whose orientation is most similar to the current tangential direction of the fiber pathway by maximizing the absolute value of their scalar product, thereby applying a simple curvature criterion. The fiber streamline $c_{i}\left(\boldsymbol{x}_{0}\right)$, which passes the $i$ th fiber bundle of the voxel containing $\boldsymbol{x}_{0}$, is then obtained by solving a curve integral on the vector field that is derived from the general fiber orientation field. This resembles fiber tractography approaches with multiple tensor models (e. g., Parker and Alexander, 2003). Further constraints are to avoid self-intersections and to minimize self-contacts of the fiber pathway, which are detected by discretizing the fiber tract over the voxel lattice using a 26-neighborhood. If appropriate, we impose the boundary condition that all nerve fibers intersect the white-gray matter interface twice. In addition, the fibers may be required to cross further brain regions.

\section{Connectivity measure}

We wish to quantify the anatomical connectivity between distant brain regions, which may not be just plain voxels (Koch et al., 2002) or single points (e. g., the center of voxels, Parker et al., 2003; Behrens et al., 2003). Therefore, the notion of source and 
target regions is generalized to (measurable) areas or volumes. Let $A$ and $B$ be two arbitrarily chosen source and target regions, respectively, with an area or volume greater than or equal to zero. Considering all the nerve fibers originating in the source region $A$, the structural connectivity may be defined in terms of the proportion of those fibers that intersect the target region $B$ while running within the brain white matter, yielding a number in the interval between zero (none of the fibers intercepts $B$ ) and one (all fibers starting in $A$ reach $B$ ). This quantity gives no information about the absolute number of connections between two regions, but reflects the degree of connectedness or the relative connection density. The connectivity of $A$ with $B$ is direction-dependent, thus generally not identical to that of $B$ with $A$ (not to be mixed up with the direction of signal exchange, i. e. afferent and efferent fibers). If the area or volume of $A$ approaches a point, this measure reduces to the existence formulation proposed by Behrens et al. (2003), which only takes values on the discrete subset $\{0,1\}$.

We draw a sample of $N$ starting points $\boldsymbol{X}_{j} \sim \mathcal{U}_{A}$ using a simple Monte Carlo approach where $\mathcal{U}_{A}$ denotes a uniform distribution on the source region $A$. The anatomical connectivity of the cortical area $A$ with a region $B$ is estimated by

$C_{A}(B) \approx \frac{1}{N} \sum_{j=1}^{N} \mathbb{J}_{B}\left(c\left(\boldsymbol{X}_{j}\right)\right)$

where $c\left(\boldsymbol{X}_{j}\right)$ is the fiber pathway with the starting point $\boldsymbol{X}_{\boldsymbol{j}}$ and $\mathbb{J}_{B}\left(c\left(\boldsymbol{X}_{j}\right)\right)$ denotes the indicator function which is 1 if $c\left(\boldsymbol{X}_{j}\right)$ passes the target region $B$ and otherwise 0 . The points in $A$ may be provided with an initial orientation $\omega$. The purpose for this constraint is to select a fiber bundle in those voxels that are composed of more than one fiber subpopulation, hence confining the subsequent connectivity analysis. The spatial density function $p_{\omega}$ encodes the volume fraction of the fiber bundle chosen by $\omega$. The sample points $\boldsymbol{X}_{j}$ are then drawn from the source region $A$ weighted by $p_{\omega}$. In the case without any information about the initial orientation, all fiber bundles are taken into account. We obtain the density function $p$ for the volume fraction of the whole fiber population on the source area $A$. Moreover, $A$ may be endowed with a regional density function $\pi_{A}$. This concept enables us to weight the interior of an area at the expense of its boundary regions, resulting in the weighted anatomical connectivity $C_{\pi_{A}}(B)$. This allows, for example, the use of Gaussian distributions to define a region of interest. In contrast, $B$ does not have any intrinsic structure. Since large populations of nerve fibers can be represented by continuous models, we might reformulate Eq. (8) by an integral.

\section{Posterior probability maps}

Bayesian statistics infers the posterior distribution of the fiber orientation field. The following algorithm summarizes the computations in order to sample the posterior distribution of the anatomical connectivity $C_{A}(B)$ of a source region $A$ (which may have an area or volume greater than zero) with a target region $B$ :

for $i:=1, \ldots, M$ do

draw a sample from the posterior distribution of the fiber orientation field;

for $j:=1, \ldots, N$ do

draw a sample point $\boldsymbol{X}_{j}$ from the source region $A$;

compute the fiber streamline $c^{(i)}\left(\boldsymbol{X}_{j}\right)$ with respect to

the $i$ th fiber orientation field sample; endfor;

\author{
compute the connectivity sample $C_{A}^{(i)}(B):=\frac{1}{N} \sum_{j=1}^{N} \mathbb{J}_{B}\left(c^{(i)}\left(\boldsymbol{X}_{j}\right)\right)$; \\ endfor.
}

This program yields a sample $\left\{C_{A}^{(i)}(B): i=1, \ldots, M\right\}$ taken from the posterior distribution $\pi\left(C_{A}(B)\right)$ of the relative connection density of $A$ with $B$. In the special case where the source and/ or target regions are formed by plain voxels, the probability distributions may be illustrated by image maps. For example, mean connectivity maps show the voxelwise expectation $\mathbb{E}_{\pi}\left[C_{A}(B)\right] \approx \frac{1}{M} \sum_{i=1}^{M} C_{A}^{(i)}(B)$. In the Bayesian analysis of functional MR data, Friston et al. (2002a,b) used posterior probability maps (PPMs) which denote the probability that the neural activation exceeds a specified threshold. We employ PPMs to depict the posterior probability $\pi\left(\vartheta \leq C_{A}(B)\right) \approx \frac{1}{M} \sum_{i=1}^{M} \mathbb{I}_{\vartheta \leq C_{A}^{(i)}(B)}$ that the anatomical connectivity of a source region with a target voxel (or the connectivity of a source voxel with a target region) is above some lower bound $\vartheta . \mathbb{I}_{\vartheta \leq C_{A}^{(i)}(B)}$ is the indicator function which is 1 if $\vartheta \leq C_{A}^{(i)}(B)$ and otherwise 0 . This enables the inference of the connectional architecture of the cerebral cortex, conditional upon the noisy MR measurements.

\section{Data acquisition and preprocessing}

The diffusion-weighted MR images are acquired by a wholebody 3 Tesla Magnetom Trio scanner (Siemens, Erlangen) equipped with an 8-channel head array coil. The spin-echo EPI sequence $(\mathrm{TE}=100 \mathrm{~ms}, \mathrm{TR}=12 \mathrm{~s}, 128 \times 128$ image matrix, FOV $=220 \times 220 \mathrm{~mm}^{2}$ ) consists of 60 diffusion encoding gradients (Jones et al., 1999) with a $b$-value of $1000 \mathrm{~s} / \mathrm{mm}^{2}$ and 7 images without any diffusion weightings, which are evenly distributed. Additionally, we employ parallel GRAPPA imaging with a reduction factor of 2 . The measurement of 72 slices with $1.7 \mathrm{~mm}$ thickness, which cover the whole brain, is repeated three times, resulting in an acquisition time of about $45 \mathrm{~min}$. A female volunteer (aged 25 years) without any known neurological disorders participated in this study. Written informed consent was obtained from the subject in accordance with the ethical approval from the University of Leipzig.

The diffusion-weighted data set is corrected for subject motion with respect to the images having a $b$-value of $0 \mathrm{~s} / \mathrm{mm}^{2}$ and is coregistered with the $T_{1}$-anatomy via a $T_{2}$-weighted MR image using rigid-body transformations (Jenkinson et al., 2002), implemented in FSL (2006). The brain is peeled from the $T_{1}$-anatomy, which was aligned with the stereotactical coordinate system proposed by Talairach and Tournoux (1988), omitting spatial normalization (Lohmann et al., 2001). After averaging, the processed diffusionweighted images are composed of three MR measurements, interpolated to the isotropic voxel resolution of $1.72 \mathrm{~mm}$.

\section{Results}

In the following, we demonstrate the reliability of the proposed forward model that links the fiber orientation distribution with the diffusion process of water molecules. We focus our attention on two nerve fiber systems, the radiation of the corpus callosum and the corticospinal tract, exemplified in a normal human brain. The callosal nerve fibers are commissural fibers which interconnect the two hemispheres, whereas the pyramidal tract is a projection fiber bundle which primarily links the motor cortex with the spinal cord. 
We are particularly interested in the region where these two nerve fiber systems cross. The water diffusion properties $\left\{\lambda_{0}, \lambda_{1}, \lambda_{2}\right\}$ of nervous tissue, which thus far has been assumed to be known, are empirically estimated. The diffusivity parameter of the isotropic compartment is set to $\lambda_{0}=0.0012 \mathrm{~mm}^{2} / \mathrm{s}$, and the apparent diffusion coefficients of a single nerve fiber are estimated at $\lambda_{1}=0.0018$ and $\lambda_{2}=0.0002 \mathrm{~mm}^{2} / \mathrm{s}$. Note that we only consider up to two fiber bundles. After a period of 5000, 10,000, and 15,000 burn-in jumps for the forward model consisting of 0,1 , and 2 fiber subpopulations, respectively, the Metropolis-Hastings algorithm (see Appendix A.3) draws 5000 samples from which every fifth is subsampled. We assume decisive evidence for the model with the larger number of fiber bundles when the Bayes factor exceeds the threshold $\xi=100$ (equal to $\ln \xi=4.6$ ). 100 randomly chosen points are used to sample each voxel of a source region. The step size of the fourth-order Runge-Kutta algorithm is the half of the isotropic voxel size, here $0.86 \mathrm{~mm}$.

\section{Simulations}

The hierarchical Bayesian model can be decomposed into subproblems. To begin with, we run simulations with 100 trials each to study the local part of the model which relates the diffusionweighted MR measurements to the underlying fiber orientation density on a voxel-by-voxel basis. The simulated MR signals are disturbed by Rician noise with the variance estimated from the image background. In the following, the spin echo signal $E_{\Delta}(\mathbf{0})$ is fixed and the volume fraction of the isotropic compartment is set to $P_{0}=0.1$. The first fiber bundle is oriented in the direction $\theta_{1}=-\pi / 4$ (latitude), $\phi_{1}=\pi / 4$ (longitude), and $\psi_{1}=\pi / 4$. The two parameters $\kappa_{1, i}=5$ and $\kappa_{3, i}=-5$ which describe the spreading of the fibers within the $i$ th bundle are fixed. The index $i$ is omitted in obvious cases.

First, we consider the parametric spherical deconvolution with one and two fiber bundles independently. In both cases, the free parameter $\kappa_{2}$ of the first fiber bundle takes different values in the interval from $\kappa_{3}$ to $\kappa_{1}$ (cf. Fig. 1). The other fiber subpopulation is rotationally symmetric and bipolar, has a volume fraction of $P_{2}=0.4$ and is oriented by $\theta_{2}=-\pi / 4$ and $\phi_{2}=-3 \pi / 8$ (corresponding to an angular separation of $72.0^{\circ}$ ). The root mean square error of the posterior mean with respect to the true parameter $\kappa_{1}-\kappa_{2}$ for the first fiber bundle is shown in the left section of Fig. 3. The posterior expectation corresponds to a Bayesian estimator with the quadratic loss function. Recall that adding any constant to $\left\{\kappa_{1}, \kappa_{2}, \kappa_{3}\right\}$ does not change the Bingham density. We note that the estimation error increases when $\kappa_{2}$ approaches $\kappa_{3}$. This can be explained by the observation that the Bingham distributions have a similar shape for a broad range of $\kappa_{2}$ near $\kappa_{3}$ (in the specific example with $\kappa_{1}=5$ and $\kappa_{3}=-5$ ). The right panel of Fig. 3 exemplifies a sample from the posterior distribution of the mean fiber direction in the case of one fiber bundle with the particular spreading $\kappa_{1}=\kappa_{2}>\kappa_{3}$. The orientation of the fiber subpopulation is shown in polar coordinates. Since the fiber direction is antipodally symmetric, it suffices to depict the orientational distribution in one hemisphere. The equatorial distribution of the samples is a benefit of the proposed reparameterization of the given problem and the adaptive Markov chain Monte Carlo method, which are addressed in more detail in Appendix A.3.

Next, we study the discrimination of multiple fiber subpopulations (Fig. 4). In the first case, the fiber orientation density is described by a Bingham distribution with the free parameter $\kappa_{2}$ in the range from $\kappa_{3}$ to $\kappa_{1}$ (cf. Fig. 1). In the second case, consider two fiber bundles whose fiber orientation density is governed by a mixture of two bipolar Watson distributions $\left(\kappa_{2, i}=\kappa_{3, i}\right)$ with equal volume fractions $\left(P_{1}=P_{2}\right)$. Their angular separation $\theta_{\text {sep }}$ varies in the range from 0 to $\pi / 2$. Fitting different models consisting of finite mixtures of $N=0,1,2$ bipolar Watson densities and $N=0,1,2$ Bingham distributions, respectively, the Bayes factor framework decides how many fiber bundles are contained in the fiber population. Fig. 4 illustrates the relative frequency of selecting the model $m_{2}$ with two fiber bundles. While the representation of the fiber orientation density by bipolar Watson distributions overestimates the number of fiber bundles, Bingham densities allow for the description of rotationally asymmetric spreading of the fibers within a bundle, but decrease the angular power to separate different fiber subpopulations.

\section{Measured data}

Fig. 5 exemplifies the posterior distribution of the mean fiber orientation in four voxels taken from the corpus callosum, the internal capsule, the crossing of the radiation of the corpus callosum

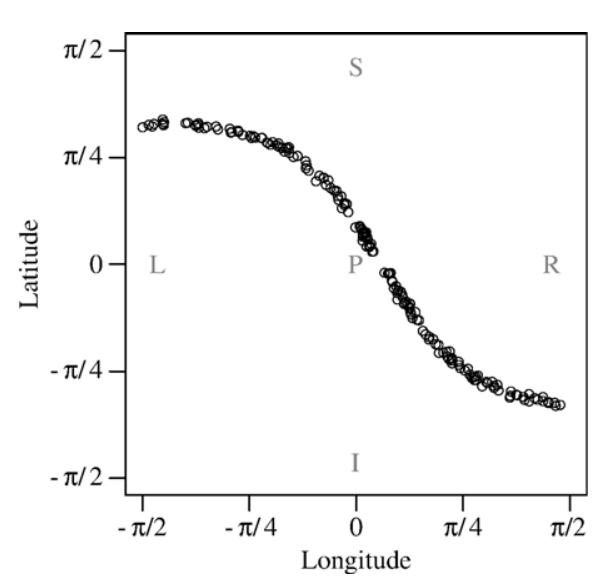

Fig. 3. The left panel shows the root mean square error of the posterior mean with respect to the true parameter $\kappa_{1}-\kappa_{2}$ which estimate the degree of the fiber spreading within the first bundle. The estimation error is displayed for simulated models with one and two fiber subpopulations. The right section depicts a sample taken from the posterior distribution of the fiber bundle direction in the case of one fiber subpopulation with $\kappa_{1}=\kappa_{2}>\kappa_{3}$. The original fiber orientation density is illustrated in Fig. 1 (right). See text for further details. 

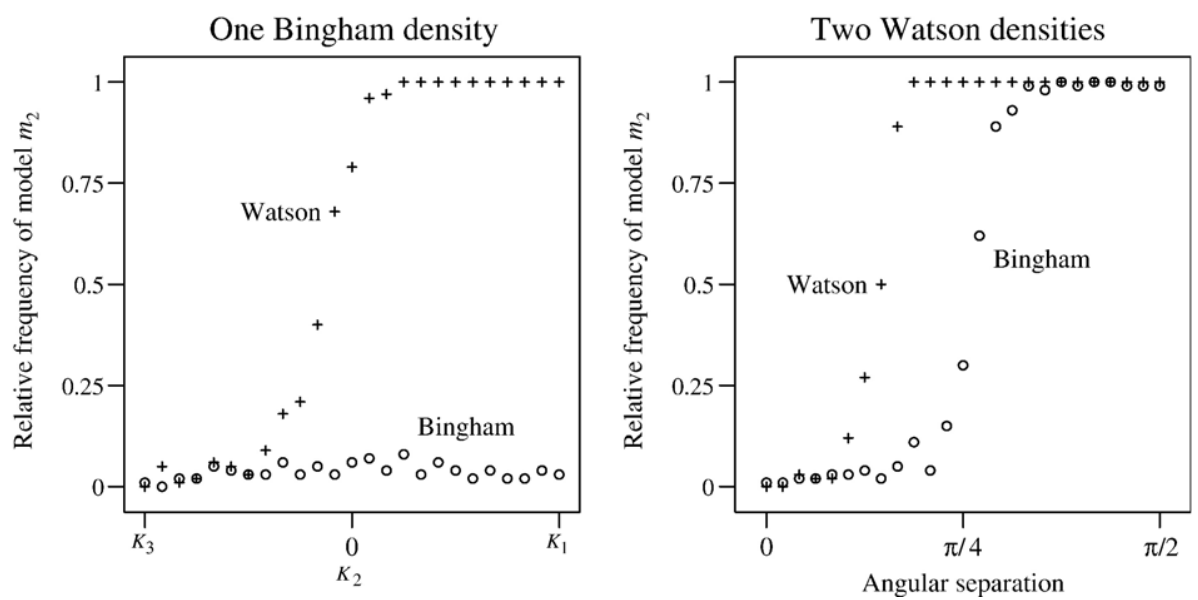

Fig. 4. Relative frequency of choosing the model $m_{2}$ with two fiber bundles assuming bipolar Watson- and Bingham-shaped fiber orientation densities, respectively. In the left section we simulate a fiber population following a Bingham distribution. The model of the right panel consists of a fiber orientation density governed by a mixture of two bipolar Watson distributions.

and the corona radiata, and the white matter region beneath the motor cortex, comparing the parametric spherical deconvolution to the well-known diffusion tensor approach (Basser et al., 1994). The Bayes factor suggests that the fiber population located in the former two brain regions consists of a single fiber bundle due to the predictive probabilities $\ln \pi\left(z \mid m_{0}\right)=-295.5, \ln \pi\left(z \mid m_{1}\right)=-209.4$, $\ln$ $\pi\left(z \mid m_{2}\right)=-207.4$ (corpus callosum) and $\ln \pi\left(z \mid m_{0}\right)=-316.2$, ln $\pi\left(z \mid m_{1}\right)=-226.6$, ln $\pi\left(z \mid m_{2}\right)=-225.7$ (internal capsule). Note that for the internal capsule the resulting distributions of the mean fiber direction are not rotationally symmetric, which is presumably due to the radiating pattern of this fiber bundle. Owing to the model probabilities $\ln \pi\left(z \mid m_{0}\right)=-271.7, \ln \pi\left(z \mid m_{1}\right)=-228.7$, and $\ln \pi\left(z \mid m_{2}\right)=$ -218.5 , there is strong evidence that the fiber population in the crossing between the radiation of the corpus callosum and the corona radiata is composed of two subpopulations. In this specific example the diffusion tensor approach yields an oblate tensor whose principal direction is equatorially distributed. (Parametric spherical deconvolution with a single fiber bundle produces similar results, which are not shown.) In the case of the region beneath the motor cortex, the chosen voxel consists of one fiber bundle due to the predictive probabilities $\ln \pi\left(z \mid m_{0}\right)=-241.9$, $\ln \pi\left(z \mid m_{1}\right)=-215.0$, and $\ln \pi\left(z \mid m_{2}\right)=-213.7$. The spreading of the fibers within this bundle is broader, as illustrated by the maximum a posteriori estimate of the fiber orientation density.

Now we demonstrate the reconstruction of the mean fiber bundle orientation in the whole diffusion-weighted data set. Fig. 6 depicts a part of the MR volume in the coronal, sagittal, and axial planes. The Bayes factor framework decides how many fiber bundles are located in each voxel. The mean direction of each fiber bundle ${ }^{1}$ is estimated through the eigenvector associated with the largest eigenvalue of the symmetric matrix $\sum_{i=1}^{N} \omega^{(i)}\left(\omega^{(i)}\right)^{t}$ where $\omega^{(i)}$ is a sample (in Cartesian coordinates) drawn from the posterior distribution of the fiber bundle orientation and $N$ denotes

\footnotetext{
${ }^{1}$ Since the mixture model formulated in the Theory and methods section is invariant with respect to the permutation of indices, the marginal distribution of the mean fiber orientation should be equal for all $N$ fiber bundles. However, this symmetry property is implicitly broken as the sampler normally fails to explore all $N$ ! modes (Jasra et al., 2005).
}

the number of samples. (If the mean fiber direction follows a Bingham distribution, this mean corresponds to its expected value.) The underlying map shows the expected fractional anisotropy provided by the Bayesian analysis of the common diffusion tensor model. Due to the adequately chosen prior distribution of the eigenvalues, the fractional anisotropy is ensured to be in the range of 0 to 1 .

Fig. 6 illustrates the brain region where the callosal fibers cross the corona radiata. The sagittal slice reveals the mean fiber bundle orientation of the corona radiata, whereas the axial plane exposes the radiation of the corpus callosum. In both cases the direction of the other fiber bundle points out of the image plane. The crossing of these two nerve fiber systems is apparent in the coronal slice. Additionally, we can find association fibers, for instance, the cingulum, the superior longitudinal fasciculus, and U-shaped fibers connecting adjacent gyri. A narrow band marked with $(*)$ causes difficulties in discretizing the fiber population into distinct subpopulations. The diffusion-weighted MR signal appears to be almost isotropic in these voxels which therefore have small fractional anisotropy values, although this region is located within the white matter reasonably far away from gray matter. We hypothesize that these voxels are composed of three fiber bundles, namely the corona radiata, the callosal fibers, and the superior longitudinal fasciculus. This is supported by simulations which show that for a $b$-value of $1000 \mathrm{~s} / \mathrm{mm}^{2}$ the macroscopic diffusion process in three orthogonal fiber subpopulations produces an MR signal that differs little from a signal of isotropic water diffusion with respect to the signal-to-noise ratio (e. g., Alexander, 2005b).

Finally, we study the connectional architecture of the pyramidal tract and the callosal fibers in Figs. 7-9. In the former case the source region is located in the internal capsule and, where necessary due to multiple fiber subpopulations at the starting point, we select the fiber bundle that is most aligned with the superior-inferior direction. The other source area is placed in the body of the corpus callosum with the initial orientation being left-right. Bayesian connectivity analysis is applied to the standard diffusion tensor approach as well as the parametric spherical deconvolution model. Figs. 7 and 8 illustrate the mean connectivity and posterior probability maps. The latter quantifies the 
Diffusion tensor model
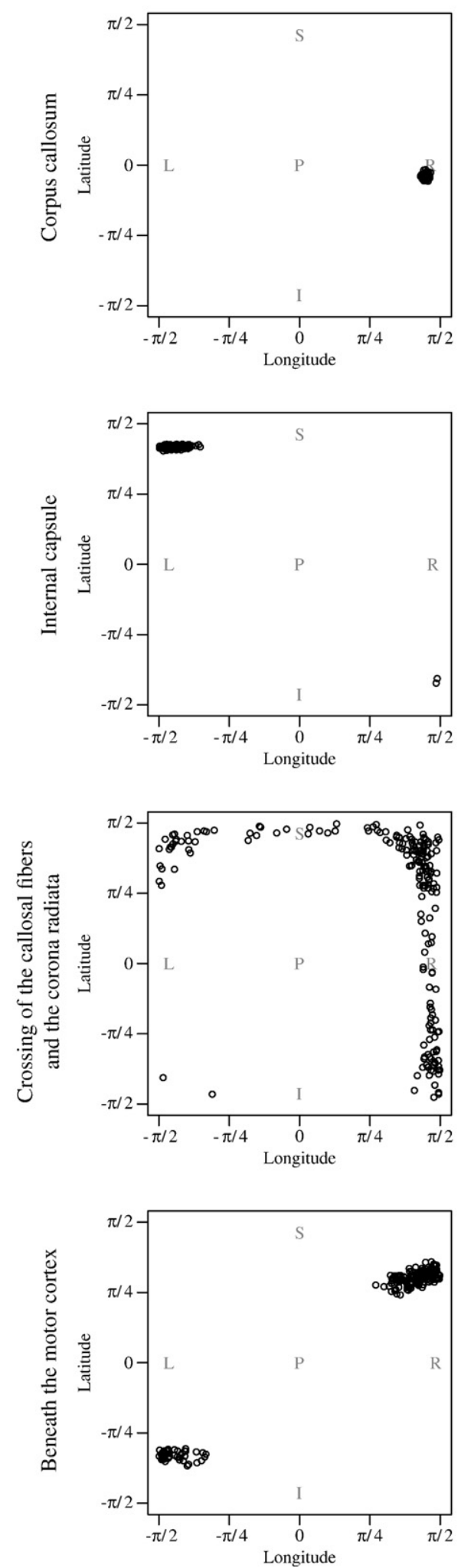

Parametric spherical deconvolution
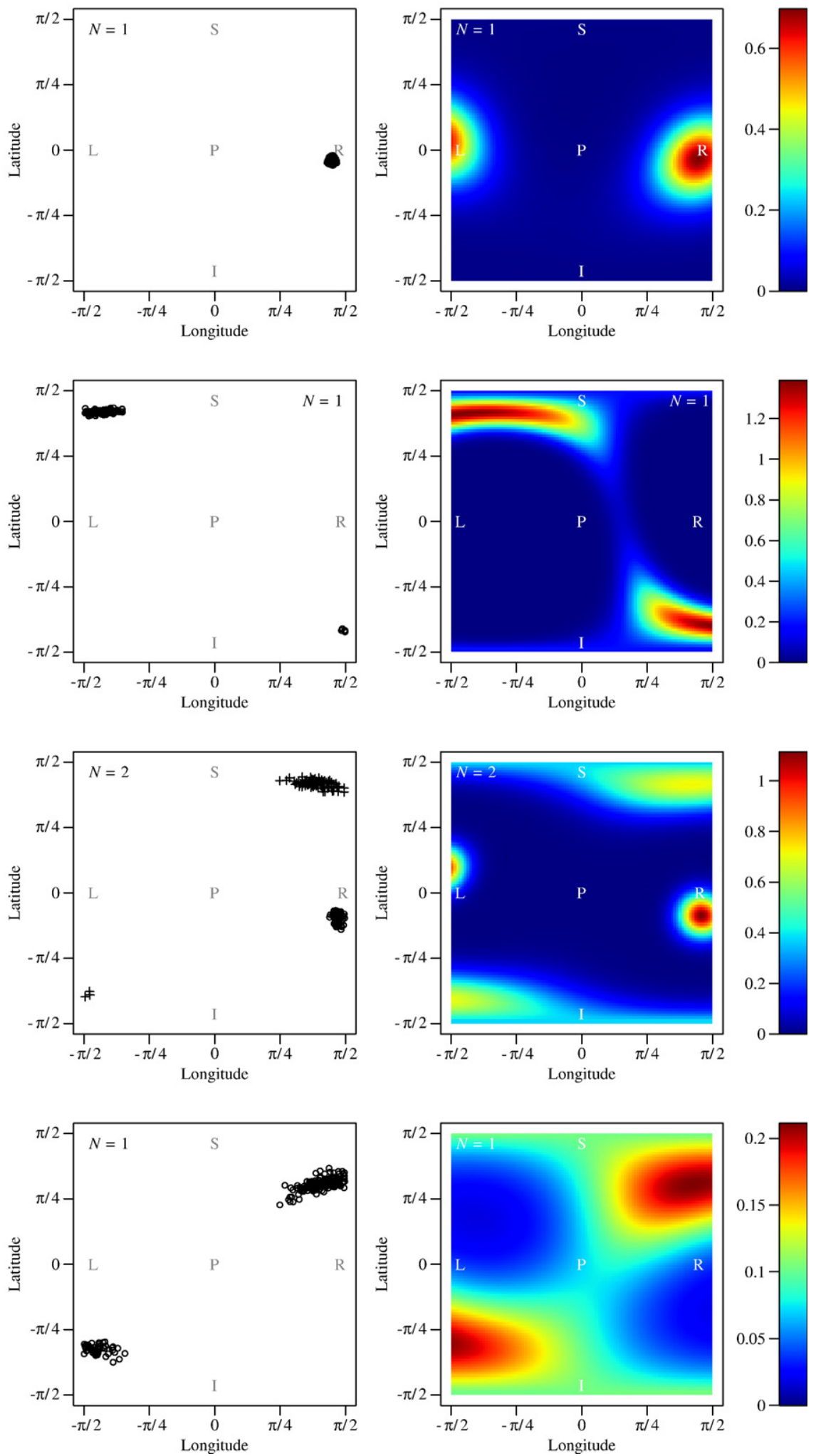
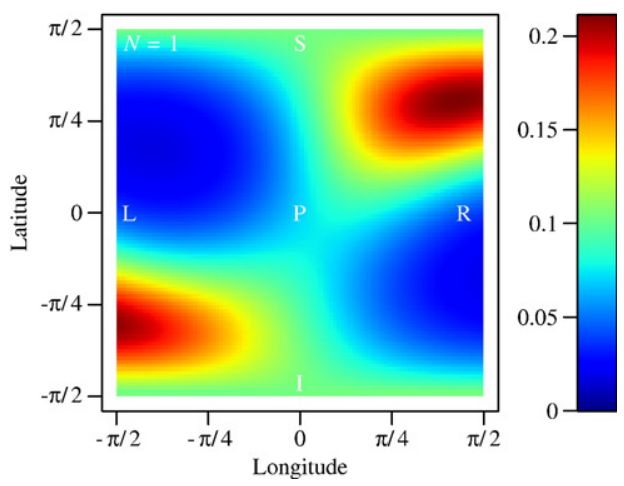

Fig. 5. Posterior distribution of the mean fiber orientation in voxels taken from four human brain regions, comparing the diffusion tensor model to the parametric spherical deconvolution. The right panel shows the maximum a posteriori estimate of the fiber orientation density. From top to bottom, the sections are the corpus callosum, the internal capsule, the crossing of the radiation of the corpus callosum and the corona radiata, and the white matter region beneath the motor cortex. The most suitable model with $N$ fiber bundles is depicted. For clarity, only 200 samples are shown. See text for further details. 

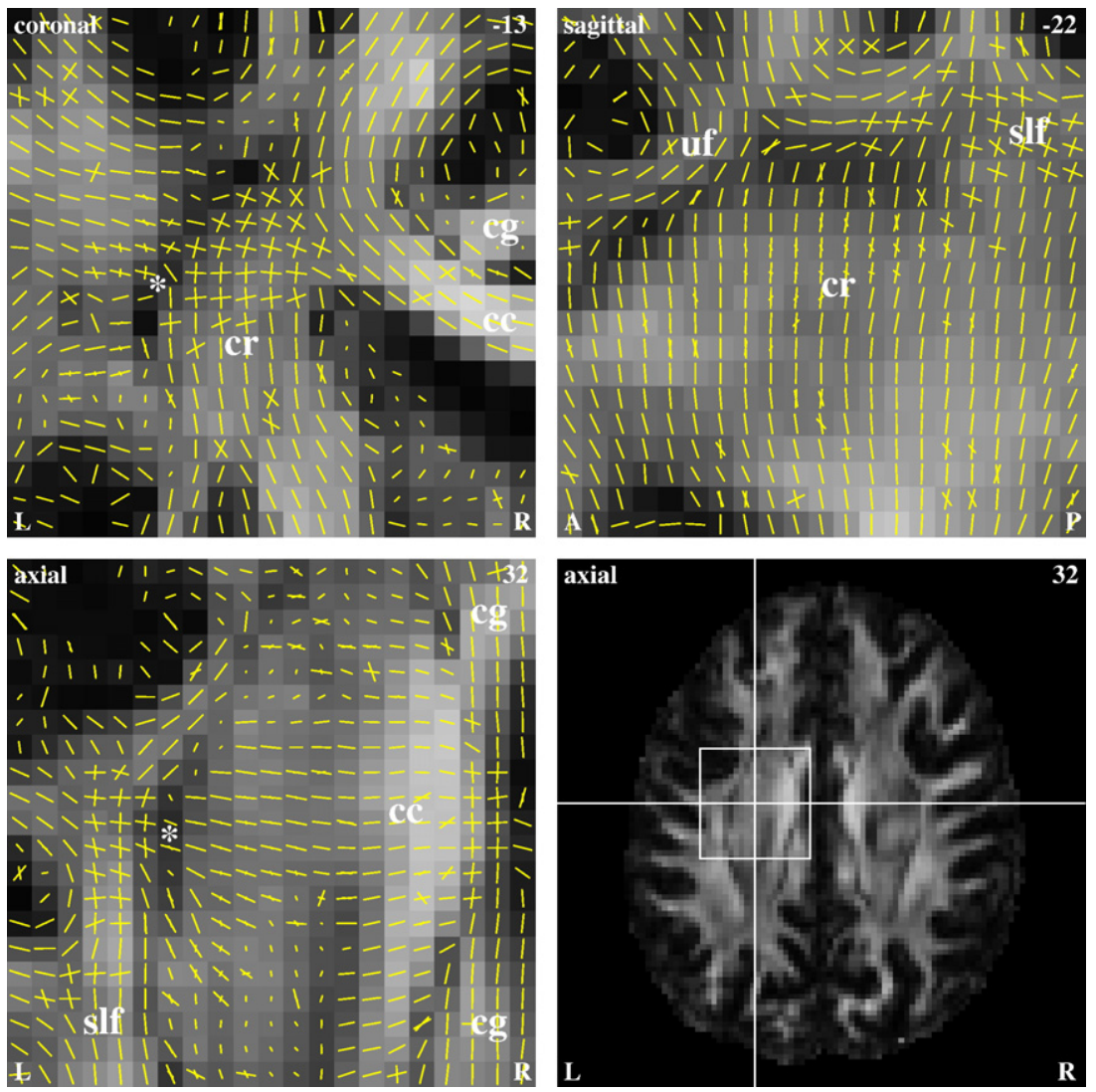

Fig. 6. Mean fiber bundle orientation of the radiation of the corpus callosum (cc), the corona radiata (cr) and their crossing. The underlying map depicts the fractional anisotropy, the crosshair position (located at the center of the coronal, sagittal and axial plot) is set at the voxel shown in the third row of Fig. 5 . Abbreviations: cingulum (cg), superior longitudinal fasciculus (slf), U-shaped association fibers (uf), approximately isotropic diffusion within the white matter (*). The number in the upper right corner indicates the slice in the stereotactical coordinate system proposed by Talairach and Tournoux (1988).

probability that at least, for instance, $10 \%$ of the fiber pathways which start in the source region pass the shown target voxel. The figures depict the maximum projection of the 10 preceding and following coronal slices of $1.72 \mathrm{~mm}$ thickness onto a reference slice, which is displayed as $T_{1}$-anatomy in the background. Fig. 9 exemplifies a sample and its cumulative density from the posterior distribution that reflects the uncertainty about the relative connection density of a source region located in the internal capsule with a target region beneath the left motor cortex.

In the case of the corticospinal tract, the connectivity maps are similar, irrespective of the underlying forward model (Fig. 7). Note that there is a presumably false positive connection of the right hemisphere via the corpus callosum in the parametric spherical deconvolution. Most remarkably, we observe different connectivity patterns for the radiation of the corpus callosum (Fig. 8). The diffusion tensor approach encounters difficulties in laterally tracking the callosal fibers, which radiate towards wide areas in both cerebral hemispheres (Pandya and Seltzer, 1986). Actually, this model suggests that the commissural fibers passing the central part of the corpus callosum mainly interconnect the left and right superior brain regions. The reason is that these nerve fibers cross the corona radiata, but the underlying model cannot represent such complex fiber populations. In contrast, the parametric spherical deconvolution enables the differentiation between fiber bundles. As shown in Fig. 8, the radiating callosal fibers also project into the lateral areas of the cerebral cortex.

\section{Discussion}

The Bayesian estimator occasionally infers connectivity values that seem, from a neuroanatomical point of view, to underestimate the degree of the connectedness of distant brain regions. While the proposed forward model allows the disentanglement of the crossing of the radiation of the corpus callosum and the corona radiata, thereby better replicating the well-known connectional neuroanatomy, it remains challenging to uncover the full radiating pattern of the interhemispheric connections via the corpus callosum. In the following, we discuss the simplifications and assumptions made to model highly complex biological systems such as the human brain white matter using diffusion MR imaging. Afterwards some perspectives for future work are highlighted.

\section{Assumptions and simplifications}

As the mapping of the microscopic tissue structure onto the diffusion process of water molecules is a projection, the inverse problem does generally not have a unique solution, thus requiring simplifying assumptions about this functional relationship. For the short diffusion times commonly used in neuroscience research and clinical domains, the second-order approximation of the water diffusion in a fiber segment by a Gaussian distribution is a sensible choice. Note that there are alternative models which may more adequately describe the diffusion process, taking the cylindrical 

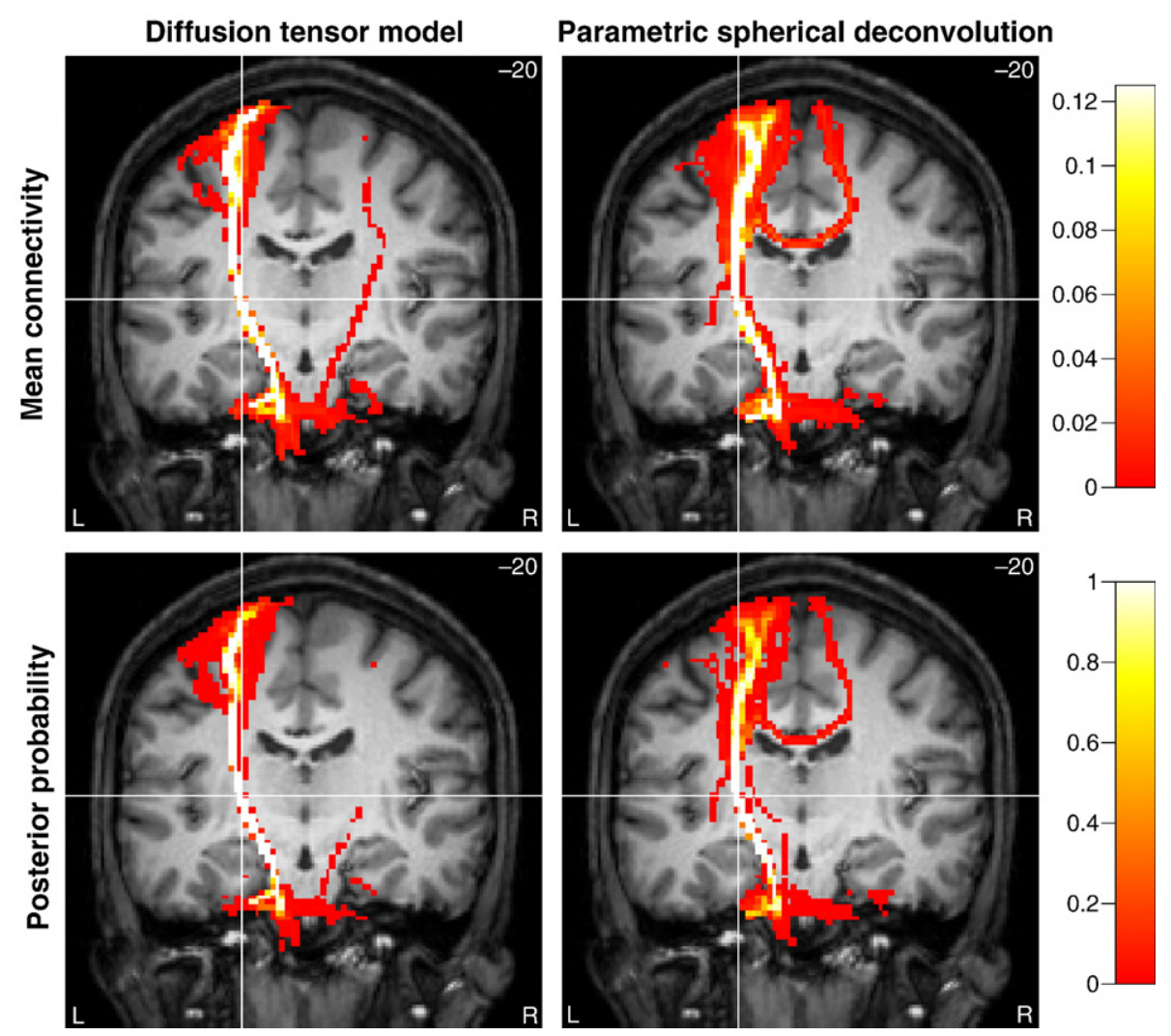

Fig. 7. Mean connectivity and posterior probability map (that the anatomical connectivity exceeds the threshold 0.1 ) of the pyramidal tract depicted in the coronal plane, comparing the diffusion tensor model with the parametric spherical deconvolution. The source region is located in the internal capsule at the position of the crosshair equivalent to the voxel illustrated in the second row of Fig. 5. In this figure and Fig. 8, connectivity or probability values below 0.002 are not shown. Mean connectivity values greater than 0.125 are white.

microgeometry of the nerve fibers into account (e. g., Söderman and Jönsson, 1995). Furthermore, it does not seem unreasonable to assume that the water exchange between the various cell components is slow with respect to the diffusion time and the permeability of the axonal membranes. Hence the water diffusion in the nerve fibers can be modeled independently.

We assumed that the unknown diffusivity parameters $\lambda_{1}$ and $\lambda_{2}$ are constant throughout the white matter, which is obviously a simplification. The axonal diameter and the degree of myelination, which both modulate the water anisotropy, vary throughout the nervous tissue. In particular, this assumption may be violated under pathological conditions where the estimation of the diffusion propagator or other derived functions (Tuch, 2004; Wedeen et al., 2005; Özarslan et al., 2006) is still reasonable. On the other hand, since the nerve fibers have similar dimensions, the proposed invariant appears to be a natural choice. Here, we empirically estimated the characteristic diffusion coefficients $\left\{\lambda_{0}, \lambda_{1}, \lambda_{2}\right\}$ of nervous tissue. A more rigorous approach would be to experimentally determine these water diffusion properties. Alternatively, we could adopt a blind deconvolution approach comprising a Bayesian model choice about $\left\{\lambda_{0}, \lambda_{1}, \lambda_{2}\right\}$. A simple Monte Carlo method tests various diffusivity values in different regions $\left\{\Omega_{i}\right\}$ of human brain white matter. These areas may be representatively selected by a neuroanatomical expert, taking advantage of prior knowledge. Then the parameter set is selected that maximizes the predictive distribution $\pi\left(z \mid \lambda_{0}, \lambda_{1}, \lambda_{2}, m_{N}\right)$ in $\left\{\Omega_{i}\right\}$.
We proposed to parameterize the fiber orientation density by a finite mixture of Bingham distributions. The components of this mixture representation describe the fiber subpopulations crossing within a voxel. Note that the modes of the fiber orientation density do, in general, not correspond to the respective directions of the fiber bundles. For example, consider a mixture of two Gaussian distributions on the real line. Depending on the difference of their means and of their variances, there may be either only one maximum or two, which, however, generally do not agree with the respective means of the two Gaussian densities. Obviously, this argument generalizes to finite mixtures of Bingham distributions. Fiber tracking along the modes of the fiber orientation density may thus introduce a systematic error.

The assumption that the arrangement of the nerve fibers within a subpopulation follows a bipolar Watson density, a particular case of the Bingham distribution (see Appendix A.1), seems to be too restrictive. The Bayes factor framework shows the strong tendency towards complex models with a large number of fiber bundles, which do not agree with the known white matter architecture. This suggests that the fibers are typically not rotationally symmetrically oriented. On the other hand, the description of the fiber orientation density by a finite mixture of Bingham distributions reduces the angular resolution for the separation of different fiber bundles. Note that the short observation period of the diffusion process, the small number of diffusion encoding gradients, the low signal-tonoise ratio, and inadequacies of the proposed model decrease the power to reveal the structural properties of the underlying material 


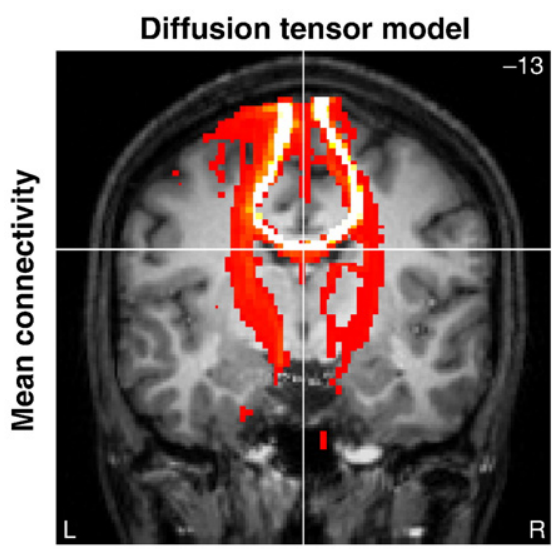

Parametric spherical deconvolution
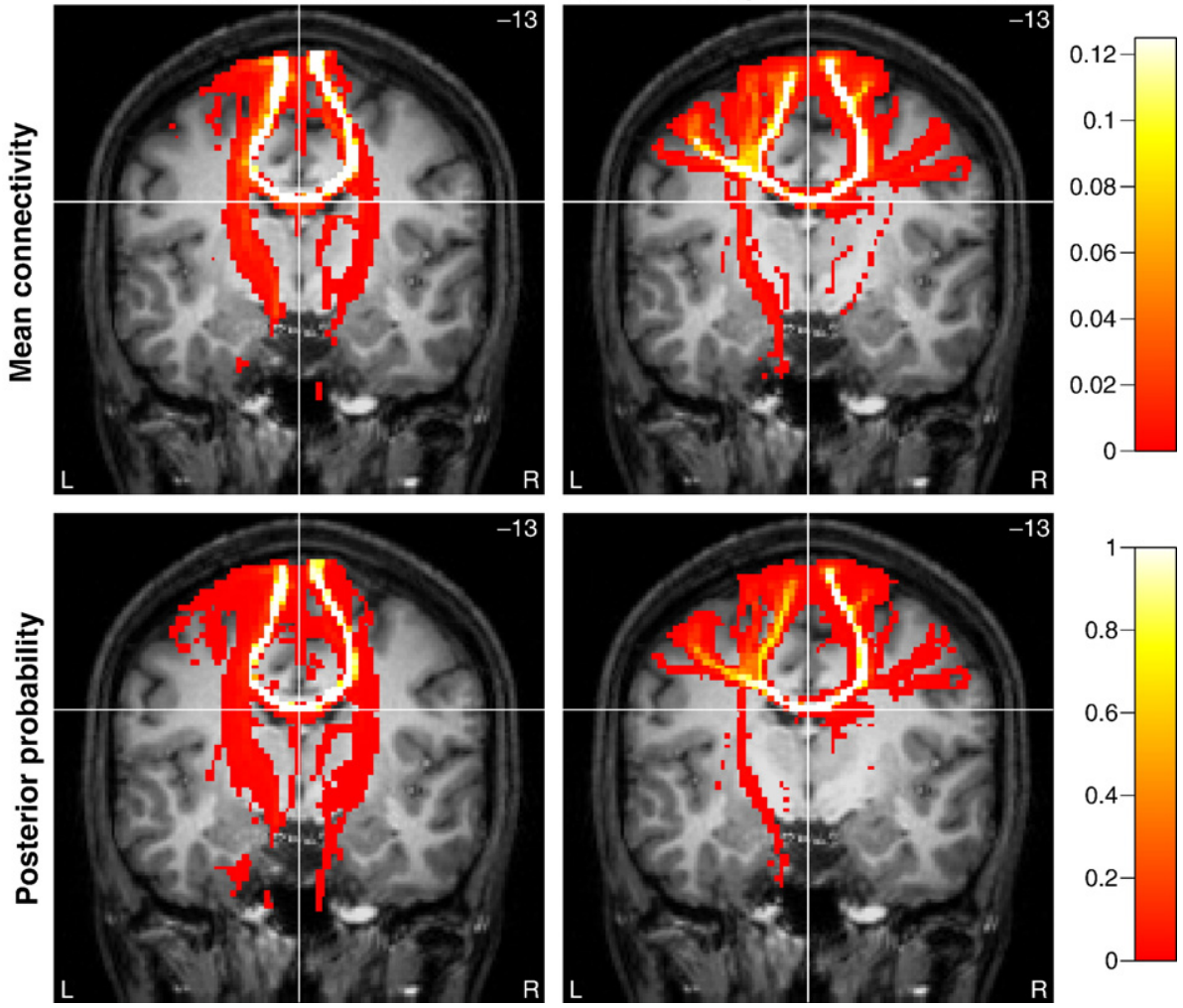

Fig. 8. Mean connectivity and posterior probability map (that the anatomical connectivity exceeds the threshold 0.1 ) of the radiation of the corpus callosum depicted in the coronal plane, comparing the diffusion tensor model with the parametric spherical deconvolution. The source region is located in the central part of the corpus callosum at the position of the crosshair equivalent to the voxel illustrated in the top row of Fig. 5.

further. The number of fiber bundles may thus be underestimated. Moreover, it would not be unreasonable to exclude the particular case where the Bingham matrix is oblate, i. e., its eigenvalues are $\kappa_{1}=\kappa_{2}>\kappa_{3}$ representing an equatorial Watson density.

The anatomical connectivity was defined as the proportion of the nerve fibers originating in a specified source region that intersect the target region, extending the idea of connectivity to arbitrarily chosen cortical areas or volumes. This quantity bears a clear neurophysiological meaning, but, taking the image modality into account, does not distinguish between afferent and efferent fibers. Nevertheless, the metric can be regarded as a simplification of a more general connectivity measure that also considers the direction of signal exchange. Note that the estimation of the measure is an approximation, since a particular fiber pathway, which does not
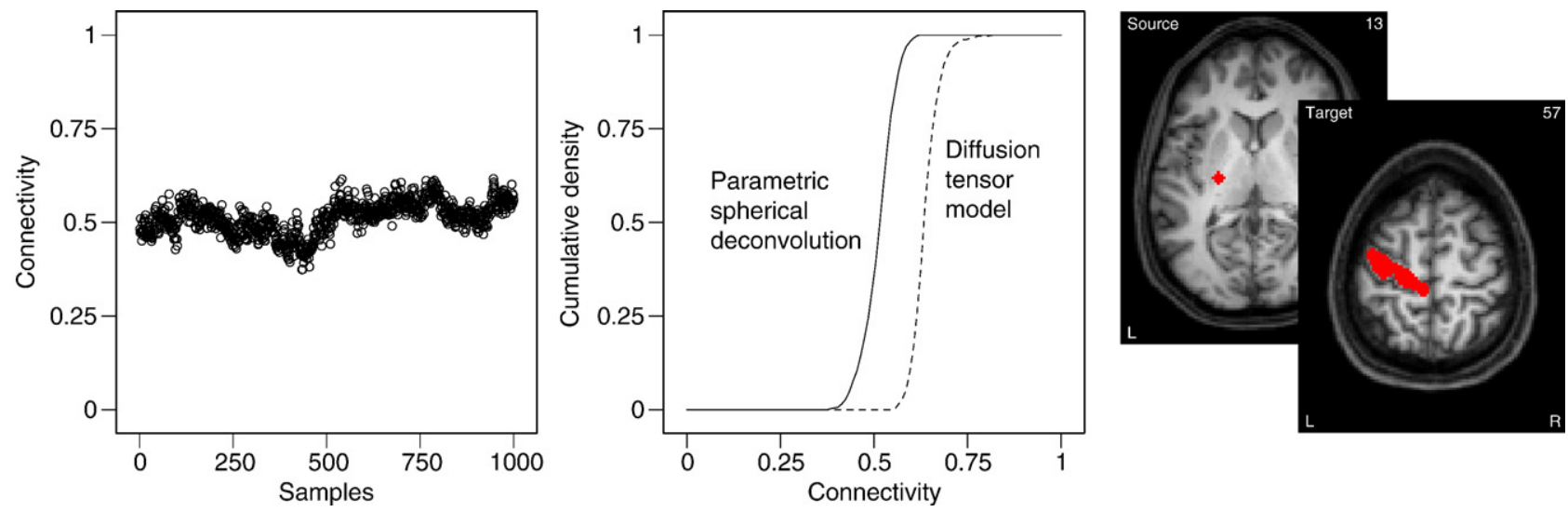

Fig. 9. Posterior probability of the anatomical connectivity of a circular area located in the internal capsule (at the position of the crosshair in Fig. 7 with a radius of $3 \mathrm{~mm}$ in the axial plane) with a target region beneath the left motor cortex, comparing the diffusion tensor model with the parametric spherical deconvolution. The left panel shows a sample drawn from the posterior distribution (only for the latter case), the middle section depicts the cumulative density functions. The parametric spherical deconvolution yields $\mathbb{E}_{\pi}\left[C_{A}(B)\right]=0.512$ and $\pi\left(C_{A}(B)>0.435 \mid z\right)=0.95$ which means that with probability 0.95 at least $43.5 \%$ of the fibers passing the source region connect the target region. 
pass the source region only once, may be considered multiple times. The posterior distribution of the structural connectivity enables us, conditional upon the observed MR measurements, to rigorously infer the connectivity patterns of the cerebral cortex in the individual living normal human brain.

\section{Perspectives}

The neural populations seem to be organized into discrete patches with respect to their connections to other brain areas via nerve fiber pathways. These homogeneous regions, uncovered by parcellating methods (Johansen-Berg et al., 2004; Anwander et al., 2007), may define the nodes of a graphical model that describes the structure of the neural network on a particular scale. Since we consider cortical (sub-) areas typically composed of several voxels, the complexity of such a graphical representation of the connectional neuroarchitecture would be greatly reduced. The interconnections of these distributed regions are denoted by directed edges endowed with the posterior probabilities on the degree of structural connectedness. This graph deals with both facets of brain organization, i. e., it enables us to infer the anatomical segregation of the cerebral cortex into structurally distinct regions and the anatomical integration of these distant areas by nerve fiber tracts. The investigation of which cortical fields are directly connected provides deep insights into functional anatomy. Possible conclusions include whether a particular brain area has a specific functional role (like primary sensory or motor cortices) or is a relay station with a great number of connections binding different cortical processing units (thereby facilitating higher brain functions). We contributed some necessary prerequisites to study these questions, which are themselves beyond the scope of this work.

\section{Acknowledgments}

The authors thank Timm Wetzel for providing the diffusionweighted MR data set. Moreover, E.K. is grateful to Gabriele Lohmann for valuable advice and fruitful discussions.

\section{Appendix A}

\section{A.1. Spherical distributions}

The Bingham distribution (Bingham, 1974) is defined as

$f_{\mathcal{B}}(\omega ; \boldsymbol{B})=\frac{1}{c_{\mathcal{B}}(\boldsymbol{B})} \exp \left(\omega^{t} \boldsymbol{B} \omega\right)$

with the 3-by-3 symmetric matrix $\boldsymbol{B}$ on the two-dimensional unit sphere $\omega \in S^{2}$. The normalizing constant is $c_{\mathcal{B}}(\boldsymbol{B})=$ $4 \pi_{1} \mathrm{~F}_{1}(1 / 2 ; 3 / 2 ; \boldsymbol{B})$ where ${ }_{1} \mathrm{~F}_{1}$ denotes the hypergeometric function of a matrix argument. A special case of the Bingham distribution is the Watson distribution (Watson, 1965)

$f_{\mathcal{W}}(\omega ; \mu, \kappa)=\frac{1}{c_{\mathcal{W}}(\kappa)} \exp \left(\kappa\langle\omega, \mu\rangle^{2}\right)$

which corresponds to $\boldsymbol{B}=\kappa \mu \mu^{t} . \mu \in S^{2}$ denotes the mean orientation. If the parameter $\kappa$ is greater than 0 , the distribution is bipolar. In the case of $\kappa ;<0, f_{\mathcal{W}}$ denotes an equatorial distribution around the axis $\mu$. If $\kappa=0$, we obtain a uniform distribution on $S^{2}$. The normalization constant is $c_{\mathcal{W}}(\kappa)=4 \pi_{1} \mathrm{~F}_{1}(1 / 2 ; 3 / 2 ; \kappa)$.

\section{A.2. Derivation of the parametric spherical deconvolution model}

Firstly, we consider the simplified diffusion propagator

$\pi(\boldsymbol{R} \mid \Delta)=\int_{S^{2}} f_{\mathcal{B}}(\omega ; \boldsymbol{B}) f_{\mathcal{N}}(\boldsymbol{R} ; \mathbf{0}, 2 \Delta \boldsymbol{D}(\omega)) d \omega$.

Inserting this diffusion propagator into the Fourier relationship formulated in Eq. (1) yields

$\frac{E_{\Delta}(\boldsymbol{q})}{E_{\Delta}(\mathbf{0})}=\int_{S^{2}} f_{\mathcal{B}}(\omega ; \boldsymbol{B})\left(\int_{\mathbb{R}^{3}} f_{\mathcal{N}}(\boldsymbol{R} ; \mathbf{0}, 2 \Delta \boldsymbol{D}(\omega)) \exp (i \boldsymbol{q} \cdot \boldsymbol{R}) \mathrm{d} \boldsymbol{R}\right) d \omega$

based on arguments in conjunction with Fubini's theorem (Rudin, 1987). Evaluating the inner integral, we obtain

$\frac{E_{\Delta}(\boldsymbol{q})}{E_{\Delta}(\mathbf{0})}=\int_{S^{2}} f_{\mathcal{B}}(\omega ; \boldsymbol{B}) \exp \left(-\Delta \boldsymbol{q}^{t} \boldsymbol{D}(\omega) \boldsymbol{q}\right) d \omega$

which is an integral equation of convolution type, since the kernel $\exp \left(-\Delta \boldsymbol{q}^{t} \boldsymbol{D}(\omega) \boldsymbol{q}\right)$ is formed by a distance function between the orientation of a fiber $\omega$ and the normalized diffusion encoding gradient direction $\boldsymbol{g}=\boldsymbol{q} /|\boldsymbol{q}|$ on the unit sphere. Substituting $\boldsymbol{D}(\omega)$ according to Eq. (2), we carry out the parametric spherical convolution

$\frac{E_{\Delta}(\boldsymbol{q})}{E_{\Delta}(\mathbf{0})}=\exp \left(-\Delta|\boldsymbol{q}|^{2} \lambda_{2}\right) \frac{{ }_{1} \mathrm{~F}_{1}\left(1 / 2 ; 3 / 2 ; \boldsymbol{B}-\Delta\left(\lambda_{1}-\lambda_{2}\right) \boldsymbol{q} \boldsymbol{q}^{t}\right)}{{ }_{1} \mathrm{~F}_{1}(1 / 2 ; 3 / 2 ; \boldsymbol{B})}$

as in Herz (1955). Finally, the full diffusion propagator (5) directly yields Eq. (6) because of the linearity of the integral operator. Let us consider two special cases. If the fibers are uniformly distributed on $S^{2}$, we get $E_{\Delta}(\boldsymbol{q}) / E_{\Delta}(\mathbf{0})=\exp \left(-\Delta|\boldsymbol{q}|^{2} \lambda_{2}\right)_{1} \mathrm{~F}_{1}\left(1 / 2 ; 3 / 2 ;-\Delta|\boldsymbol{q}|^{2}\right.$ $\left.\left(\lambda_{1}-\lambda_{2}\right)\right)$. If all fibers within a chosen population have the same orientation $\omega$, we obtain the diffusion tensor model $E_{\Delta}(\boldsymbol{q}) / E_{\Delta}(\mathbf{0})=$ $\exp \left(-\Delta \boldsymbol{q}^{t} \boldsymbol{D}(\omega) \boldsymbol{q}\right)$ where the diffusivity parameters $\lambda_{1}$ and $\lambda_{2}$ are fixed.

\section{A.3. Computational issues of Bayesian statistics}

The Metropolis-Hastings algorithm (Metropolis et al., 1953; Hastings, 1970) explores the high-dimensional space of the posterior distribution $\pi\left(\theta \mid z, m_{N}\right)$ by local random walk moves with the step size $\Sigma$. For efficiency reasons we fix the non-diagonal entries of the matrix $\Sigma$ to zero. As a result, the convergence properties of the sampler worsen. In particular, the estimation of the parameters representing the fiber bundle orientations degenerates, since these random variables are generally far from being independent. Therefore, we reparameterize the directional parameters by applying the rotation $\boldsymbol{R}_{i}^{-1}$ on the $i$ th fiber subpopulation. The rotation matrix arises from the eigen-decomposition of the Bingham matrix $\boldsymbol{B}_{i}=\boldsymbol{R}_{i} \operatorname{diag}\left(\kappa_{1, i}, \kappa_{2, i}, \kappa_{3, i}\right) \boldsymbol{R}_{i}^{-1}$. This coordinate transformation reduces the interdependencies of the orientational parameters. If there is only one fiber bundle, we can obtain a reasonable approximation of the rotation matrix from the estimation of the diffusion tensor model by linear regression.

The covariance matrix $\Sigma$ of the random walk determines the speed at which the simulated Markov chain reaches its stationary regime. If the moves are small, the acceptance probability is high and the random walk explores the distribution only slowly. If the step size is large, the acceptance rate is low and it takes a long time for the sampler to converge to the posterior distribution. The 
objective is to find the optimal scaling $\Sigma$ for each individual voxel (Roberts et al., 1997) because $\Sigma$ is not known due to our limited knowledge about the posterior distribution before sampling. Therefore, the step size $\Sigma$ of the random walk is adapted during the period of burn-in (Haario et al., 2001; Andrieu and Robert, 2001). Note that we limit the entries of the matrix $\Sigma$ which represent the orientations of the nerve fiber bundles by setting the maximum variance to $\pi / 2$. This stochastic optimization by the Robbins-Monro algorithm (Robbins and Monro, 1951) does not only calculate the asymptotic variance, but also the asymptotic mean of the Euler angles $\phi_{i}, \theta_{i}$, and $\psi_{i}$ of the $i$ th fiber subpopulation. The inverse of the rotation matrix $\boldsymbol{R}_{i}$ which is determined from these mean angular values reparameterizes the directional parameters as described above.

The random-walk Metropolis-Hastings algorithm converges to the target distribution irrespective of the starting point. For example, the initial volume fraction $P_{0}$ of the extra-axonal compartment minimizes $\left(\bar{S}_{\Delta,|\boldsymbol{q}|} / \bar{S}_{\Delta, \mathbf{0}}-\left[P_{0} \exp \left(-\Delta|\boldsymbol{q}|^{2} \lambda_{0}\right)+\left(1-P_{0}\right)\right.\right.$ $\left.\left.\exp \left(-\Delta|\boldsymbol{q}|_{2} \lambda_{2}\right)_{1} \mathrm{~F}_{1}\left(1 / 2 ; 3 / 2 ;-\Delta|\boldsymbol{q}|^{2}\left(\lambda_{1}-\lambda_{2}\right)\right)\right]\right)^{2}$ in the range from 0 to 1 where $\bar{S}_{\Delta, 0}$ and $\bar{S}_{\Delta,|q|}$ denote the mean of the measured MR signals ( $\Delta$ fixed) in the absence of any diffusion weightings and with $0<|\boldsymbol{q}|=$ const, respectively. However, the high dimensionality of the multiple fiber bundle problem and the heterogeneous scaling of the various model parameters slow down the convergence rate of the sampler. To accelerate the initial transient phase, the Watson density is temporarily used to constrain the arrangement of the nerve fibers within a bundle to be rotationally symmetric. In addition, we employ a coarse-to-fine approach and smooth the noisy MR measurements by a spherical spline model (Wahba, 1981), thus reducing the number of modes in the posterior distribution. After the Markov chain has approached the region of high probability using the smoothed signals, the sampler continues with the original MR data set.

\section{References}

Alexander, D.C., 2005a. Maximum entropy spherical deconvolution for diffusion MRI. Proceedings of the 19th International Conference on Information Processing in Medical Imaging, pp. 76-87.

Alexander, D.C., 2005b. Multiple-fiber reconstruction algorithms for diffusion MRI. Ann. N. Y. Acad. Sci. 1064, 113-133.

Anderson, A.W., 2005. Measurement of fiber orientation distributions using high angular resolution diffusion imaging. Magn. Reson. Med. 54, 1194-1206.

Andrieu, C., Robert, C.P., 2001. Controlled memc for optimal sampling. Tech. rep., Université Paris-Dauphine.

Anwander, A., Tittgemeyer, M., von Cramon, D.Y., Friederici, A.D., Knösche, T.R., 2007. Connectivity-based parcellation of Broca's area. Cereb. Cortex 17, 816-825.

Basser, P.J., Jones, D.K., 2002. Diffusion-tensor MRI: theory, experimental design and data analysis-A technical review. NMR Biomed. 15, $456-467$.

Basser, P.J., Pajevic, S., 2000. Statistical artifacts in diffusion tensor MRI (DT-MRI) caused by background noise. Magn. Reson. Med. 44, 41-50.

Basser, P.J., Mattiello, J., Le Bihan, D., 1994. Estimation of the effective selfdiffusion tensor from the NMR spin echo. J. Magn. Reson., Ser. B $103,247-254$

Beaulieu, C., 2002. The basis of anisotropic water diffusion in the nervous system-A technical review. NMR Biomed. 15, 435-455.

Behrens, T.E.J., Woolrich, M.W., Jenkinson, M., Johansen-Berg, H., Nunes, R.G., Clare, S., Matthews, P.M., Brady, J.M., Smith, S.M., 2003. Characterization and propagation of uncertainty in diffusion-weighted MR imaging. Magn. Reson. Med. 50, 1077-1088.
Bingham, C., 1974. An antipodally symmetric distribution on the sphere. Ann. Stat. 2, 1201-1225.

Callaghan, P.T., 1993. Principles of Nuclear Magnetic Resonance Microscopy. Oxford Univ. Press, Oxford.

Conturo, T.E., Lori, N.F., Cull, T.S., Akbudak, E., Snyder, A.Z., Shimony, J.S., McKinstry, R.C., Burton, H., Raichle, M.E., 1999. Tracking neuronal fiber pathways in the living human brain. Proc. Natl. Acad. Sci. U. S. A. 96, 10422-10427.

Cook, P.A., Alexander, D.C., Parker, G.J.M., 2004. Modelling noiseinduced fibre-orientation error in diffusion-tensor MRI. Proceedings of the 2nd IEEE International Symposium on Biomedical Imaging: From Macro to Nano, pp. 332-335.

Cook, P.A., Zhang, H., Avants, B.B., Yushkevich, P., Alexander, D.C., Gee, J.C., Ciccarelli, O., Thompson, A.J., 2005. An automated approach to connectivity-based partitioning of brain structures. Proceedings of the 8th International Conference on Medical Image Computing and Computer-Assisted Intervention, Part I, pp. 164-171.

Einstein, A., 1905. Über die von der molekularkinetischen Theorie der Wärme geforderten Bewegung von in ruhenden Flüssigkeiten suspendierten Teilchen. Ann. Phys. 17, 549-560.

Friman, O., Farnebäck, G., Westin, C.-F., 2006. A Bayesian approach for stochastic white matter tractography. IEEE Trans. Med. Imag. 25, 965-978.

Friston, K.J., Glaser, D.E., Henson, N.A., Kiebel, S., Phillips, C., Ashburner, J., 2002a. Classical and Bayesian inference in neuroimaging: applications. NeuroImage 16, 484-512.

Friston, K.J., Penny, W., Phillips, C., Kiebel, S., Hinton, G., Ashburner, J., 2002b. Classical and Bayesian inference in neuroimaging: theory. NeuroImage 16, 465-483.

FSL, 2006. FMRIB Software Library. University of Oxford. URL http:// www.fmrib.ox.ac.uk/fsl.

Haacke, E.M., Brown, R.W., Thompson, M.R., Venkatesan, R., 1999. Magnetic Resonance Imaging: Physical Principles and Sequence Design. Wiley-Liss, New York.

Haario, H., Saksman, E., Tamminen, J., 2001. An adaptive metropolis algorithm. Bernoulli 7, 223-242.

Hastings, W.K., 1970. Monte Carlo sampling methods using Markov chains and their applications. Biometrika 57, 97-109.

Healy, D.M., Kim, P.T., 1996. An empirical Bayes approach to directional data and efficient computation on the sphere. Ann. Stat. 24, 232-254.

Herz, C.S., 1955. Bessel functions of matrix arguments. The Annals of Mathematics 61, 474-523.

Hosey, T., Williams, G., Ansorge, R., 2005. Inference of multiple fiber orientations in high angular resolution diffusion imaging. Magn. Reson. Med. 54, 1480-1489.

Jansons, K.M., Alexander, D.C., 2003. Persistent angular structure: new insights from diffusion magnetic resonance imaging data. Inverse Problems 19, 1031-1046.

Jasra, A., Holmes, C.C., Stephens, D.A., 2005. Markov chain Monte Carlo methods and the label switching problem in bayesian mixture modeling. Stat. Sci. 20, 50-67.

Jeffreys, H., 1961. Theory of Probability. Oxford Univ. Press, New York.

Jenkinson, M., Bannister, P., Brady, M., Smith, S., 2002. Improved optimization for the robust and accurate linear registration and motion correction of brain images. NeuroImage 17, 825-841.

Johansen-Berg, H., Behrens, T.E.J., Robson, M.D., Drobnjak, I., Rushworth, M.F.S., Brady, J.M., Smith, S.M., Higham, D.J., Matthews, P.M., 2004. Changes in connectivity profiles define functionally distinct regions in human medial frontal cortex. Proc. Natl. Acad. Sci. U. S. A. 101, $13335-13340$.

Jones, D.K., Horsfield, M.A., Simmons, A., 1999. Optimal strategies for measuring diffusion in anisotropic systems by magnetic resonance imaging. Magn. Reson. Med. 42, 515-525.

Kass, R.E., Raftery, A.E., 1995. Bayes factors. J. Am. Stat. Assoc. 90, 773-795.

Koch, M.A., Norris, D.G., Hund-Georgiadis, M., 2002. An investigation of 
functional and anatomical connectivity using magnetic resonance imaging. NeuroImage 16, 241-250.

Kume, A., Wood, A.T.A., 2005. Saddlepoint approximations for the Bingham and Fisher-Bingham normalising constants. Biometrika 92, 465-476.

Le Bihan, D., 2003. Looking into the functional architecture of the brain with diffusion MRI. Nat. Rev., Neurosci. 4, 469-480.

Lohmann, G., Müller, K., Bosch, V., Mentzel, H., Hessler, S., Chen, L., Zysset, S., von Cramon, D.Y., 2001. Lipsia-A new software system for the evaluation of functional magnetic resonance images of the human brain. Comput. Med. Imaging Graph. 25, 449-457.

Marin, J.-M., Mengersen, K., Robert, C.P., 2005. Bayesian modelling and inference on mixtures of distributions. Handbook of Statistics: Bayesian Thinking, Modeling and Computation, vol. 25. Elsevier, pp. 459-507.

Metropolis, N., Rosenbluth, A.W., Rosenbluth, M.N., Teller, A.H., 1953. Equation of state calculations by fast computing machines. J. Chem. Phys. 21, 1087-1092.

Mori, S., Crain, B.J., Chacko, V.P., van Zijl, P.C.M., 1999. Threedimensional tracking of axonal projections in the brain by magnetic resonance imaging. Ann. Neurol. 45, 265-269.

Newton, M.A., Raftery, A.E., 1994. Approximate Bayesian inference with the weighted likelihood bootstrap. J. R. Stat. Soc., Ser. B Stat. Methodol. 56, 3-48.

Özarslan, E., Shepherd, T.M., Vemuri, B.C., Blackband, S.J., Mareci, T.H., 2006. Resolution of complex tissue microarchitecture using the diffusion orientation transform (dot). NeuroImage 31, 1086-1103.

Pandya, D.N., Seltzer, B., 1986. The topography of commissural fibers. Neurology and Neurobiology. Two Hemispheres-One Brain: Functions of the Corpus Callosum. Sixth International Symposium of the Center for Research in Neurological Sciences, Montreal, Canada, 1984, vol. 17, pp. 47-74.

Parker, G.J.M., Alexander, D.C., 2003. Probabilistic Monte Carlo based mapping of cerebral connections utilising whole-brain crossing fibre information. Proceedings of the 18th International Conference on Information Processing in Medical Imaging, pp. 684-695.

Parker, G.J.M., Haroon, H.A., Wheeler-Kingshott, C.A.M., 2003. A framework for a streamline-based probabilistic index of connectivity (pico) using a structural interpretation of MRI diffusion measurements. J. Magn. Reson. Imaging 18, 242-254.

Robbins, H., Monro, S., 1951. A stochastic approximation method. Ann. Math. Stat. 22, 400-407.

Roberts, G.O., Gelman, A., Gilks, W.R., 1997. Weak convergence and optimal scaling of random walk metropolis algorithms. Ann. Appl. Probab. 7, 110-120.

Rudin, W., 1987. Real and Complex Analysis. McGraw-Hill, New York.

Sijbers, J., den Dekker, A.J., 2004. Maximum likelihood estimation of signal amplitude and noise variance from $\mathrm{mr}$ data. Magn. Reson. Med. 51, 586-594.

Söderman, O., Jönsson, B., 1995. Restricted diffusion in cylindrical geometry. J. Magn. Reson., Ser. A 117, 94-97.

Sporns, O., Tononi, G., Edelman, G.M., 2000. Theoretical neuroanatomy: relating anatomical and functional connectivity in graphs and cortical connection matrices. Cereb. Cortex 10, 127-141.

Stejskal, E.O., Tanner, J.E., 1965. Spin diffusion measurements: spin echoes in the presence of a time-dependent field gradient. J. Chem. Phys. 42, 288-292.

Talairach, J., Tournoux, P., 1988. Co-Planar Stereotaxic Atlas of the Human Brain. Thieme, Stuttgart

Tournier, J.-D., Calamante, F., Gadian, D.G., Connelly, A., 2004. Direct estimation of the fiber orientation density function from diffusionweighted MRI data using spherical deconvolution. NeuroImage 23, 1176-1185.

Tuch, D.S., 2004. Q-ball imaging. Magn. Reson. Med. 52, 1358-1372.

Tuch, D.S., Reese, T.G., Wiegell, M.R., Makris, N., Belliveau, J.W., Wedeen, V.J., 2002. High angular resolution diffusion imaging reveals intravoxel white matter fiber heterogeneity. Magn. Reson. Med. 48, 577-582.

Wahba, G., 1981. Spline interpolation and smoothing on the sphere. SIAM J. Sci. Statist. Comput. 2, 5-16.

Watson, G.S., 1965. Equatorial distribution on a sphere. Biometrika 52, 193-201. Wedeen, V.J., Hagmann, P., Tseng, W.-Y.I., Reese, T.G., Weisskoff, R.M., 2005. Mapping complex tissue architecture with diffusion spectrum magnetic resonance imaging. Magn. Reson. Med. 54, 1377-1386.

Zeki, S., Shipp, S., 1988. The functional logic of cortical connections. Nature 335, 311-317. 